\title{
Stochastic Lifestyling: Optimal Dynamic Asset Allocation for Defined Contribution Pension Plans
}

\author{
Andrew J.G. Cairns ${ }^{\text {ab }}$, David Blake ${ }^{\mathrm{c}}$, Kevin Dowd ${ }^{\mathrm{d}}$ \\ First version: June 2000 \\ This version: September 13, 2004
}

\begin{abstract}
This paper considers the asset-allocation strategies open to members of definedcontribution pension plans. We investigate a model that incorporates three sources of risk: asset risk and salary (or labour-income) risk in the accumulation phase; and interest-rate risk at the point of retirement. We propose a new form of terminal utility function, incorporating habit formation, that uses the plan member's final salary as a numeraire. The paper discusses various properties and characteristics of the optimal stochastic asset-allocation strategy (which we call stochastic lifestyling) both with and without the presence of non-hedgeable salary risk. We compare the performance of stochastic lifestlying with some popular strategies used by pension providers, including deterministic lifestyling (which involves a gradual switch from equities to bonds according to preset rules) and static strategies that invest in benchmark mixed funds. We find that the use of stochastic lifestyling significantly enhances the welfare of a wide range of potential plan members relative to these other strategies.
\end{abstract}

JEL classification: E21, G11, G23.

Keywords: stochastic control; optimal asset allocation; stochastic lifestyling; utility numeraire; habit formation; non-hedgeable salary risk; HJB equation.

\footnotetext{
${ }^{a}$ Actuarial Mathematics and Statistics, School of Mathematical and Computer Sciences, Heriot-Watt University, Edinburgh, EH14 4AS, United Kingdom

${ }^{\mathrm{b}}$ Corresponding author: E-mail A.Cairns@ma.hw.ac.uk

${ }^{\mathrm{c} C}$ Cass Business School, City University, 106 Bunhill Row, London, EC1Y 8TZ, United Kingdom

${ }^{\mathrm{d}}$ Centre for Risk \& Insurance Studies, Nottingham University Business School, Jubilee Campus, Nottingham, NG8 1BB, United Kingdom
} 


\section{Introduction}

A popular asset allocation strategy for managing equity risk during the accumulation phase of a defined contribution (DC) pension plan is deterministic lifestyling. At the beginning of the plan, the contributions are invested entirely in equities. Then, beginning on a predetermined date (e.g., ten years) prior to retirement, the assets are switched gradually into bonds at a rate equal to the inverse of the length of the switchover period (e.g., 10\% per year). By the date of retirement, all the assets are held in bonds, which are then sold to purchase a life annuity that provides the pension. The aims of the strategy are to reduce the impact on the pension of a catastrophic fall in the stock market just before the plan member retires and to hedge the interest-rate risk inherent in the annuity-purchase decision. Deterministic lifestyling is a simple strategy to explain to plan members and to implement, and is widely used as the default strategy or as one option offered by many UK DC pensions providers. Similar deterministic strategies have also been recommended in other countries (for example, in a US context, Malkiel, 2003, recommends a mix of bonds and equities which changes over time in a similar way to the deterministic lifestyle strategy). However, there is no evidence that it is an optimal strategy in an objective sense.

The purpose of this paper is to find the optimal dynamic asset allocation strategy for a defined contribution pension plan, taking into account the stochastic features of the plan member's lifetime salary progression as well as the stochastic properties of the assets held in his accumulating pension fund. Of particular importance is the fact that salary risk (or labour-income risk: the fluctuation in the plan member's earnings in response to economic shocks) is not fully hedgeable using existing financial assets. To illustrate, wage-indexed bonds could be used to hedge both productivity and inflation shocks, but such bonds are not widely traded. The paper builds on Blake, Cairns \& Dowd (2001) which developed a pension plan accumulation programme designed to deliver a retirement pension that is closely related to the salary (and hence standard of living) that the plan member received immediately prior to retirement. We call the optimal dynamic asset allocation strategy stochastic lifestyling and compare it against various static and deterministic lifestyle strategies to calculate the cost of suboptimal strategies. Moreover, stochastic lifestyling is still a relatively easy strategy to implement in practice, despite the apparent increase in complexity compared to deterministic lifestyling.

The solution technique uses the expected present value of future contribution premiums into the plan. This is not a new idea and has been used by Boulier et al. (2001), Deelstra et al. (2000) and Korn \& Krekel (2002) and others, building on the original work of Merton $(1969,1971)$. Liu (2001) examines ways in which the Merton framework can be generalised to include, for example, stochastic interest rates and stochastic risk premia, but only for the case where utility is a function 
of the cash lump sum at the beginning of retirement.

Where our approach differs from these studies is in:

- the use of a salary-related numeraire (or utility numeraire) as an argument in the plan member's utility function ${ }^{1}$; and

- assuming that the purpose of the pension plan is to deliver a pension (i.e. life annuity) in retirement rather than a cash lump sum at the date of retirement $^{2}$.

Although these differences do not alter the basic form of the optimal solution derived in these earlier studies, we find that the optimal proportions invested in each of the key asset classes, cash, bonds and equities, are very different. More significantly, we also find that these optimal proportions often differ substantially from those implied by deterministic lifestyling (which ignores both the plan member's attitude to risk and any correlation between his salary and the returns on assets held in the fund), so that the cost of the latter strategy can be considerable in terms of the additional premiums into the plan needed to match the expected utility of the optimal strategy.

However, unlike the case for deterministic lifestyling, the optimal asset allocation under stochastic lifestyling is sensitive to certain underlying assumptions, e.g., concerning the process determining interest rates. To clarify key issues, we therefore first derive our results using a simple stochastic model in which the interest rate is deterministic (Section 2). We then extend the model to a more general stochastic setting (Section 3). This allows us to analyse separately (a) the effect of the salary-related numeraire in the utility function and (b) the pension purchased at retirement and its dependence on uncertain interest rates.

We show that in the former case the optimal asset allocation can be replicated using two efficient mutual funds, whereas the latter case needs three efficient mutual funds. One mutual fund (which is heavily dominated with equities) is designed to satisfy the risk appetite of the plan member. The second fund (which is heavily dominated with cash) is designed to hedge the salary risk within the pension plan. The third fund (which is heavily dominated with bonds) is designed to hedge interest rate (and hence annuity) risk in the case where interest rates are stochastic. $^{3}$

\footnotetext{
${ }^{1}$ In the studies of Boulier et al., 2001, and Deelstra et al., 2000, utility is also measured relative to a salary-related benchmark, but the utility function depends on the monetary surplus over this benchmark, rather than on the surplus as a proportion of final salary as here.

${ }^{2}$ In countries, such as the UK, it is mandatory to use the cash lump sum to purchase a life annuity by a certain age, while in other countries, such as the US, it is not.

${ }^{3}$ Parts of the problem are related to previous analyses of defined-benefit (DB) pension plans (Sundaresan and Zapatero, 1997, and Cairns, 2000). Both the DC and DB problems require the
} 


\section{A simple stochastic model}

\subsection{The structure of the model}

This section develops the optimal asset allocation strategy using a simple model with deterministic nominal interest rates. The aim of the simple model is to highlight the following features:

- the use of the plan member's salary as a numeraire in the utility function;

- the treatment of a stream of contribution premiums linked to salary; and

- the consequences of salary not being fully hedgeable.

The first two features are straightforward to deal with and do not cause particular problems when salary is fully hedgeable (that is, when there is a complete market). The third feature, however, implies that the market is incomplete and so gives rise to qualitatively different results from the complete market case.

The structure of the simple model is as follows:

- There are two underlying assets in which the pension plan can invest: one risk free (a cash fund) and one risky (an equity fund). The risk-free asset has a price $R_{0}(t)=R_{0}(0) \exp (r t)$ at time $t$, where $r$ is the constant risk-free nominal rate of interest. The risky asset has price $R_{1}(t)$ at time $t$ and satisfies the stochastic differential equation $(\mathrm{SDE}) d R_{1}(t)=R_{1}(t)\left[\left(r+\xi_{1} \sigma_{1}\right) d t+\sigma_{1} d Z_{1}(t)\right]$, where $Z_{1}(t)$ is a standard Brownian motion and $\xi_{1}$ and $\sigma_{1}$ are constants. The risk premium on this asset is $\xi_{1} \sigma_{1}$, where $\xi_{1}$ is the market price of risk. The solution for $R_{1}(t)$ is

$$
R_{1}(t)=R_{1}(0) \exp \left[\left(r+\xi_{1} \sigma_{1}-\frac{1}{2} \sigma_{1}^{2}\right) t+\sigma_{1} Z_{1}(t)\right] .
$$

- The pension plan member has a salary at time $t$ of $Y(t) . Y(t)$ is governed by the SDE $d Y(t)=Y(t)\left[\left(r+\mu_{Y}\right) d t+\sigma_{Y 0} d Z_{0}(t)+\sigma_{Y 1} d Z_{1}(t)\right]$, where $\mu_{Y}$ is a constant and $Z_{0}(t)$ is a second standard Brownian motion independent of $Z_{1}(t)$. The $\sigma_{Y 1}$ term allows for possible correlation between the salary

determination of an optimal asset-allocation strategy using stochastic control methods. However, typical DB problems involve optimising the sponsor's utility, whereas here we are optimising the individual plan member's utility. The DB model of Sundaresan and Zapatero, 1997, shares some characteristics with the DC models of Deelstra et al. (2000) and Boulier et al. (2001). These three papers incorporate a DB type of guarantee, and all three analyses rely upon salary risk being fully hedgeable. However, in the DB plan surplus over the guarantee reverts to the plan sponsor while in the DC plans the full value of the assets goes to the member. 
and equity returns (for example, salary might be related to the profitability of the company or the general state of the economy). $Y(t)$ has the solution

$$
\begin{aligned}
Y(t) & =Y_{H}(t) Y_{N}(t) \\
\text { where } Y_{H}(t) & =Y(0) \exp \left[\left(r+\mu_{Y}-\frac{1}{2} \sigma_{Y 1}^{2}\right) t+\sigma_{Y 1} Z_{1}(t)\right] \\
\text { and } Y_{N}(t) & =\exp \left[-\frac{1}{2} \sigma_{Y 0}^{2}+\sigma_{Y 0} Z_{0}(t)\right] .
\end{aligned}
$$

$Y_{H}(t)$ is the hedgeable component of $Y(t)$ and $Y_{N}(t)$ is the non-hedgeable component.

- Premiums are payable in one of two forms: either (a) as regular premiums, i.e., continuously into the plan member's individual account at the rate of $\pi Y(t)$, so that premiums are a constant proportion, $\pi$, of salary ${ }^{4}$, or (b) as an initial single premium, in which case $\pi=0$.

- The value of the plan member's account (i.e., his pension wealth) is denoted by $W(t)$. If a (possibly time-dependent) proportion $p(t)$ of this account is invested in the risky asset then the dynamics of $W(t)$ are governed by the SDE

$$
d W(t)=W(t)\left[\left(r+p(t) \xi_{1} \sigma_{1}\right) d t+p(t) \sigma_{1} d Z_{1}(t)\right]+\pi Y(t) d t .
$$

- The plan member will retire at time $T$, is risk averse, and has a terminal utility function, $u(W(T), Y(T))$, that depends both on terminal pension wealth and terminal income

$$
u(W(T), Y(T))= \begin{cases}\frac{1}{\gamma}\left(\frac{W(T)}{Y(T)}\right)^{\gamma} & \text { where } \gamma<1 \text { and } \gamma \neq 0 \\ \log \left(\frac{W(T)}{Y(T)}\right) & \text { when } \gamma=0 .^{5}\end{cases}
$$

- The plan member's objective is to find the optimal dynamic asset allocation strategy, namely the weight in equities, $p(t)$, to maximise his expected terminal utility.

\footnotetext{
${ }^{4}$ The contribution rate $\pi$ is given exogeneously, as is typical in many occupational DC plans. Also implicit in this model is the assumption that the rate of consumption before retirement is given exogenously and is equal to $(1-\pi) Y(t)$; in other words, there are no non-pensions savings in the model.

${ }^{5}$ In the case of $\log$ utility, $E[\log W(T) / Y(T)]=E[\log W(T)]-E[\log Y(T)]$. Since $E[\log Y(T)]$ is fixed, the optimal investment strategy will be the same for this case as for problems where the utility function is just $\log W(T)$ (i.e., the utility function involves just a cash numeraire).
} 
The new numeraire is straightforward to justify. At the time of retirement, the plan member will be concerned about the preservation of his standard of living. So he will be interested in his retirement income relative to his pre-retirement salary. This is consistent with consumption-smoothing features of the life-cycle model of Ando and Modigliani (1963). It is also consistent with the habit-formation model developed by Ryder and Heal (1973), Sundaresan (1989) and Constantinides (1990). Specifically, the plan member's utility at retirement is directly related to his or her recent salary, $Y(T)$, or consumption level, given exogenously as $(1-\pi) Y(T)$.

Defining a new state variable $X(t) \equiv W(t) / Y(t)$ allows terminal utility to be written $u(X(T))=\gamma^{-1} X(T)^{\gamma}$. A straightforward application of Ito's formula shows that the SDE for $X(t)$ is

$$
\begin{aligned}
d X(t)=[\pi+X(t) & \left.\left(-\mu_{Y}+p(t) \sigma_{1}\left(\xi_{1}-\sigma_{Y 1}\right)+\sigma_{Y 0}^{2}+\sigma_{Y 1}^{2}\right)\right] d t \\
& -\sigma_{Y 0} X(t) d Z_{0}(t)+X(t)\left(p(t) \sigma_{1}-\sigma_{Y 1}\right) d Z_{1}(t) .
\end{aligned}
$$

From equation (2.1.3) we can see that $X(t)$ provides us with all the information we require to solve the problem. Additional information on $W(t)$ and $Y(t)$ will not alter the ultimate distribution for $X(T)$. In the remainder of this section, therefore we will focus on $X(t)$ alone.

The optimisation problem can now be stated as: Maximise, over all admissible asset allocation stategies, the expected terminal utility (that is: maximise, over $p(t), E[u(X(T))])$.

We will now consider four separate cases:

- Case 1: $\pi=0, \sigma_{Y 0}=0$.

- Case 2: $\pi=0, \sigma_{Y 0} \neq 0$.

- Case 3: $\pi>0, \sigma_{Y 0}=0$.

- Case 4: $\pi>0, \sigma_{Y 0} \neq 0$.

The first two cases indicate zero contributions to the pension fund, and would apply to a plan member who has accumulated some pension wealth and has chosen not to save any more towards retirement. The latter two cases indicate positive ongoing contributions. The first and the third cases indicate zero non-hedgeable salary risk and the second and fourth cases indicate the existence of non-hedgeable salary risk. 


\subsection{Case 1: $\pi=0, \sigma_{Y 0}=0$, and Case 2: $\pi=0, \sigma_{Y 0} \neq 0$}

We will first consider Case 2. Case 1 can then be solved by taking $\sigma_{Y 0}=0$. In Case 2, the salary numeraire cannot be replicated with existing financial assets, since the market is incomplete. Nevertheless we can still derive an analytical solution to the problem. The optimal expected utility (the value function) is

$$
V(t, X(t))=h(t) X(t)^{\gamma}
$$

where

$$
\begin{aligned}
h(t) & =\frac{1}{\gamma} \exp \left[\gamma \theta_{2}(T-t)\right] \\
\text { and } \theta_{2} & =\frac{\gamma+1}{2} \sigma_{Y 0}^{2}+\frac{\left(\xi_{1}-\sigma_{Y 1}\right)^{2}}{2(1-\gamma)}+\xi_{1} \sigma_{Y 1}-\mu_{Y} .
\end{aligned}
$$

The associated optimal asset allocation strategy involves a constant equity weight

$$
p(t, X(t))=p^{*} \equiv \frac{\sigma_{Y 1}}{\sigma_{1}}+\frac{\xi_{1}-\sigma_{Y 1}}{(1-\gamma) \sigma_{1}}, \text { for all } t .
$$

These results indicate that, although $\sigma_{Y 0}$ does have an impact on the expected terminal utility, the optimal asset allocation strategy is unaffected by the size of $\sigma_{Y 0}$. In other words there is nothing that the plan member can do to offset the effect of unhedgeable volatility in his salary: he just has to accept its existence. At first sight, this conclusion might appear inconsistent with the theory of background risk. Pratt and Zeckhauser (1987), Kimball (1993) and Gollier and Pratt (1996) argue that the introduction of background risk should make risk-averse investors even more risk averse. However, this does not happen here for two reasons. First, when $\pi=0$, the investor's pension wealth, $W(t)$, is unaffected by the background risk (here, non-hedgeable salary risk) since there are no future premiums. In contrast, the key arguments in background risk theory assume that $W(t)$ is affected by the background risk. Second, the background risk only appears in the terminal utility function through the numeraire. From equation (2.1.2) we recall that $Y(T)$ is the product of its non-hedgeable (background risk) and hedgeable components. Since these are independent and since we are using power utility, we can separate the expected utility into a product of two expectations. It follows that the existence of non-hedgeable salary risk (when $\pi=0$ ) has no impact on the optimisation problem. We shall see below that when $\pi>0$ the existence of non-hedgeable salary risk does result in a lower investment in equities.

The optimal value function for Case 1 is found simply by setting $\sigma_{Y 0}=0$ and the optimal asset allocation strategy is still given by equation (2.2.1).

In both these cases, the fact that the optimal equity weight is unchanging throughout the life of the accumulation programme indicates that lifestyling, whether deterministic or stochastic, cannot be the optimal strategy. 


\subsection{Case 3: $\pi>0, \sigma_{Y 0}=0$}

Most DC pension plans involve an ongoing stream of contribution premiums. This leads to a significant change in the optimal asset allocation strategy. In Case 3, the future premiums can be fully hedged, that is, a future payment of $\pi Y(t)$ can be replicated exactly using a combination of cash and equities, as can terminal salary, $Y(T)$. In such circumstances, the market is complete and we can attach a unique price to the stream of future premiums. The market price at time $t$ for the premiums payable between $t$ and $T$ (i.e., their discounted value) can be written as

$$
\begin{aligned}
E_{Q}\left[\int_{t}^{T} e^{-r(s-t)} \pi Y(s) d s \mid \mathcal{F}_{t}\right] & =\pi Y(t) f(t) \\
\text { where } f(t) & =\frac{\exp \left[\left(\mu_{Y}-\xi_{1} \sigma_{Y 1}\right)(T-t)\right]-1}{\mu_{Y}-\xi_{1} \sigma_{Y 1}} .
\end{aligned}
$$

In this equation, $\mathcal{F}_{t}$ is the filtration (or information) generated by $Z_{0}(u)$ and $Z_{1}(u)$ up to time $t$, and $E_{Q}$ implies expectation with respect to the unique risk-neutral measure $Q$ rather than the real-world measure. ${ }^{6}$

This market price is the key to the solution of Case 3, because it enables us to treat the promised future premiums as if they were part of the current assets of the pension plan

$$
\tilde{W}(t) \equiv W(t)+Y(t) \pi f(t) .
$$

We refer to $\tilde{W}(t)$ as the augmented pension wealth (see Boulier et al, 2001, and Deelstra et al, 2000).

Market completeness also allows us to construct a synthetic asset or mutual fund, $R_{2}(t)$, whose dynamics are governed by the SDE

$$
d R_{2}(t)=R_{2}(t)\left[\left(r+\xi_{1} \sigma_{Y 1}\right) d t+\sigma_{Y 1} d Z_{1}(t)\right] .
$$

This mutual fund can be used to hedge perfectly the stream of future contribution premiums, since it is perfectly correlated with salary risk.

Let $q(t)$ represent the proportion of augmented pension wealth, $\tilde{W}(t)$, invested at time $t$ in $R_{1}(t)$, with the remainder invested in $R_{2}(t)$. The holding in $R_{2}(t)$ therefore comprises (a) a short holding of $-\pi f(t) Y(t)$ which will be repaid completely from future premiums, and (b) a positive holding equal to $(1-q(t)) \tilde{W}(t)$. We can show, by application of Ito's formula, that

$$
d \tilde{W}(t)=\tilde{W}(t)\left[q(t)\left(\left(r+\xi_{1} \sigma_{1}\right) d t+\sigma_{1} d Z_{1}(t)\right)+(1-q(t))\left(\left(r+\xi_{1} \sigma_{Y 1}\right) d t+\sigma_{Y 1} d Z_{1}(t)\right)\right] .
$$

\footnotetext{
${ }^{6}$ Under $Q, d R_{1}(t)=R_{1}(t)\left[r d t+\sigma_{1} d \tilde{Z}_{1}(t)\right]$ and $d Y(t)=Y(t)\left[\left(r+\mu_{Y}-\xi_{1} \sigma_{Y 1}\right) d t+\sigma_{Y 1} d \tilde{Z}_{1}(t)\right]$, where $\tilde{Z}_{1}(t)$ is a standard $Q$-Brownian motion.
} 
Now consider $\tilde{X}(t) \equiv \tilde{W}(t) / Y(t)=X(t)+\pi f(t)$. Note that the terminal utility can be expressed in terms of $\tilde{X}(T)$ as $u(\tilde{X}(T))=\gamma^{-1} \tilde{X}(T)^{\gamma}$. We can show that

$d \tilde{X}(t)=\tilde{X}(t)\left[\left(\xi_{1} \sigma_{Y 1}-\mu_{Y}+q(t)\left(\xi_{1}-\sigma_{Y 1}\right)\left(\sigma_{1}-\sigma_{Y 1}\right)\right) d t+q(t)\left(\sigma_{1}-\sigma_{Y 1}\right) d Z_{1}(t)\right]$

and the optimal expected terminal utility is

$$
\begin{aligned}
V(t, X(t)) & =h(t)(X(t)+\pi f(t))^{\gamma} \\
\text { where } h(t) & =\frac{1}{\gamma} \exp \left[\gamma \theta_{3}(T-t)\right] \\
\text { and } \theta_{3} & =\frac{\left(\xi_{1}-\sigma_{Y 1}\right)^{2}}{2(1-\gamma)}+\xi_{1} \sigma_{Y 1}-\mu_{Y} .
\end{aligned}
$$

It follows that the optimal expected utility differs from Case 1 only because of the inclusion of the present value of the future premiums.

Using $V(t, X(t))$, the optimal value for $q(t)$ is

$$
q^{*}(t, \tilde{X}(t))=q^{*} \equiv \frac{\xi_{1}-\sigma_{Y 1}}{(1-\gamma)\left(\sigma_{1}-\sigma_{Y 1}\right)} .
$$

Therefore, the amount of pension wealth invested in equities expressed in units of $Y(t)$ is

$$
X(t)\left(q^{*}+\left(1-q^{*}\right) \frac{\sigma_{Y 1}}{\sigma_{1}}\right)+\pi f(t) \frac{\left(\sigma_{1}-\sigma_{Y 1}\right)}{\sigma_{1}} q^{*},
$$

and the optimal proportion invested in equities is

$$
p^{*}(t, X(t))=\left(q^{*}+\left(1-q^{*}\right) \frac{\sigma_{Y 1}}{\sigma_{1}}\right)+\frac{\pi f(t)}{X(t)} \frac{\left(\sigma_{1}-\sigma_{Y 1}\right)}{\sigma_{1}} q^{*} .
$$

The relationship between these two time-varying components means that $p^{*}(t, X(t))$ exhibits what appears to be traditional lifestyle dynamics: that is, it starts high (provided $\left.\left(\sigma_{1}-\sigma_{Y 1}\right) q^{*} / \sigma_{1}>0\right)$ and gradually drifts lower as $f(t)$ decreases and $X(t)$ increases. However, this downward drift is stochastic rather than deterministic and falls to $q^{*}+\left(1-q^{*}\right) \sigma_{Y 1} / \sigma_{1}$ rather than to 0 as under traditional deterministic lifestyling. Hence, the optimal asset-allocation strategy can be described as stochastic lifestyling.

We note that stochastic lifestyling is easy to implement in this case, since the only time-varying components of $p^{*}(t, X(t))$ are (a) the deterministic term $f(t)$ and (b) the stochastic diffusion $X(t)$.

If we employ the optimal asset allocation strategy then

$$
\begin{aligned}
X(T) & =\tilde{X}(T) \\
& =\tilde{X}(0) \exp \left[\left(\xi_{1} \sigma_{Y 1}-\mu_{Y}+\frac{\left(\xi_{1}-\sigma_{Y 1}\right)^{2}(1-2 \gamma)}{2(1-\gamma)^{2}}\right) T+\frac{\left(\xi_{1}-\sigma_{Y 1}\right)}{(1-\gamma)} Z_{1}(T)\right]
\end{aligned}
$$


with $\tilde{X}(0) \equiv X(0)+\pi f(0)$.

To illustrate, consider the following set of parameters (which are broadly compatible with UK data over the last century $)^{7}$

$$
\mu_{Y}=0, \quad \xi_{1}=0.2, \quad \sigma_{1}=0.2, \quad \sigma_{Y 1}=0.05, \quad \pi=0.1, \quad T=20
$$

The optimal equity mix is illustrated in Figure 1 which shows the dependence of the optimal equity proportion and amount, respectively, on $X$ when $T-t=$ 20. We can see for low values of $X(0)$ that the optimal equity mix is far from its asymptotic value of $q^{*}+\left(1-q^{*}\right) \sigma_{Y 1} / \sigma_{1}$. For completeness, Figure 1 also shows the optimal strategy if net wealth, $W(t)$, is allowed to become negative. In practice, negative net wealth might not be an interesting case to consider. However, within the context of the present model (complete market, no constraints on short selling), many strategies can be followed (including the optimal one) that could allow net wealth to fall to as low as $-\pi f(t)$. The completeness of the market means that we can always follow a strategy that will guarantee that the fund will be positive by time $T$. In reality most sample paths will be positive for all but the earliest years of the plan ${ }^{8}$.

Figure 2 shows $p^{*}(t, X(t))$ in two simulations. ${ }^{9}$ In path $B$, values are higher because $X(t)$ is lower and close to 0 for the first few years, causing greater volatility. Both paths clearly show the operation of stochastic lifestyling: the optimal equity proportion varies in a stochastic way from year to year, although it will gradually decline because both $t$ and (in general) $X(t)$ will be increasing. This is why we refer to the optimal strategy as stochastic lifestyling. In contrast, deterministic lifestyling adopts a more risky asset allocation to begin with before shifting in a pre-determined way over, say, the final 10 years out of equities into bonds. Further, with stochastic lifestyling, the equity proportion declines to a non-zero level which depends on both the plan member's degree of risk aversion and the degree of correlation with the plan member's salary. By comparison, the equity proportion falls to zero with deterministic lifestyling, irrespective of the plan member's degree of risk aversion or salary dynamics (see path $\mathrm{C}$ in Figure 2).

\footnotetext{
${ }^{7}$ Setting $\mu_{Y}=0$ was considered reasonable since long-term average salary increases are similar to average long-run interest rates. A reasonable value for $r$ would be 0.06 (nominal), but its value is, in the present model, irrelevant. $\pi$ can be set to 0.1 without loss of generality because we are using power utility. Any other value could be used for $\pi$ but there would be no impact on the optimal strategy or on how it compares with other strategies.

${ }^{8}$ Just after commencement of the plan, when $X=0$, if the fund has gone short in some assets, the Brownian motion will cause sample paths for $X(t)$ to dip slightly below 0 for short periods of time.

${ }^{9}$ The basic shapes of these paths are consistent with the intuition provided by Malkiel (2003) who states that older plan members "have fewer years of labor income ahead of them. Thus they cannot count on salary income to sustain them should the stock market have a period of negative returns. ... Hence, stocks should comprise a smaller proportion of their assets." What is new here is the stochastic nature of $p^{*}(t, X(t))$.
} 

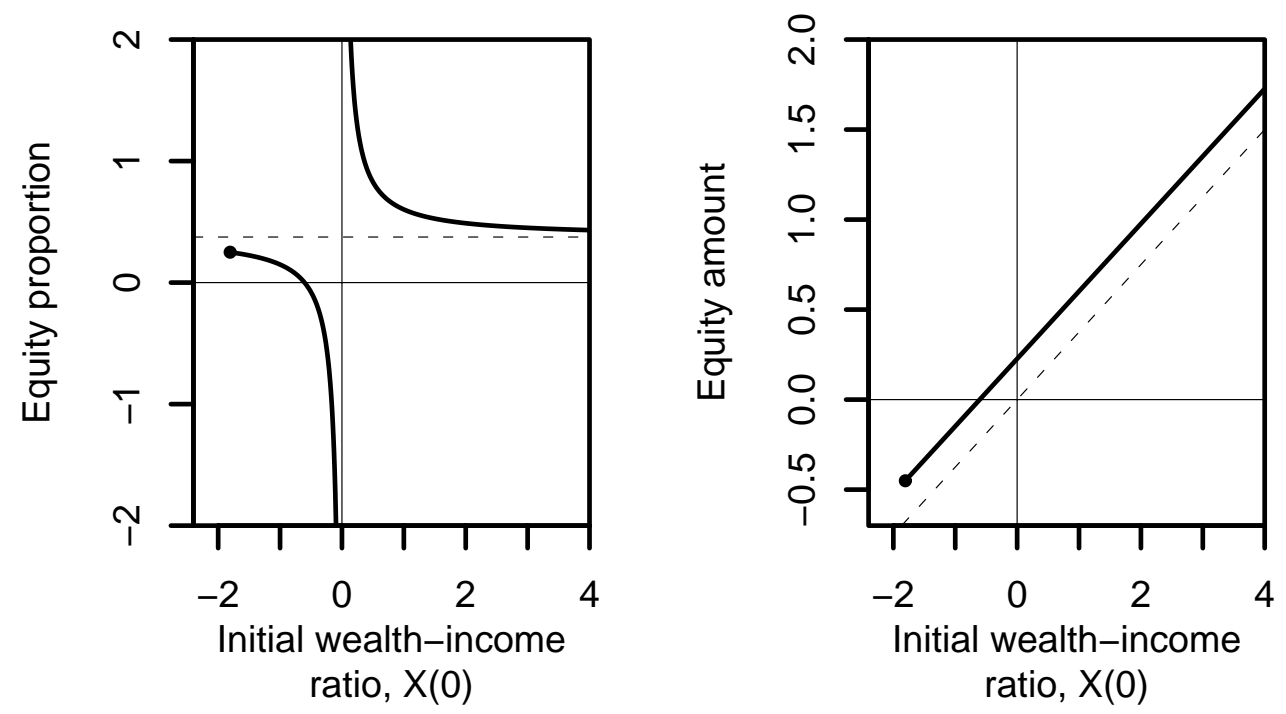

Figure 1: Left: optimal equity proportion, $p^{*}(0, X(0))$. Right: optimal equity amounts, $X(0) p^{*}(0, X(0))$, expressed in units of $Y(t)$ when relative risk aversion is $6(\gamma=-5)$ and $T=20$. Parameters are $\mu_{Y}=0, \xi_{1}=0.2, \sigma_{1}=0.2, \sigma_{Y 1}=$ $0.05, \pi=0.1$. The asymptotic value for $p^{*}(0, X(0))$ as $X(0) \rightarrow \infty$ is 0.375 (dotted line). The dot on the left indicates the lower limit on $X(0)$.

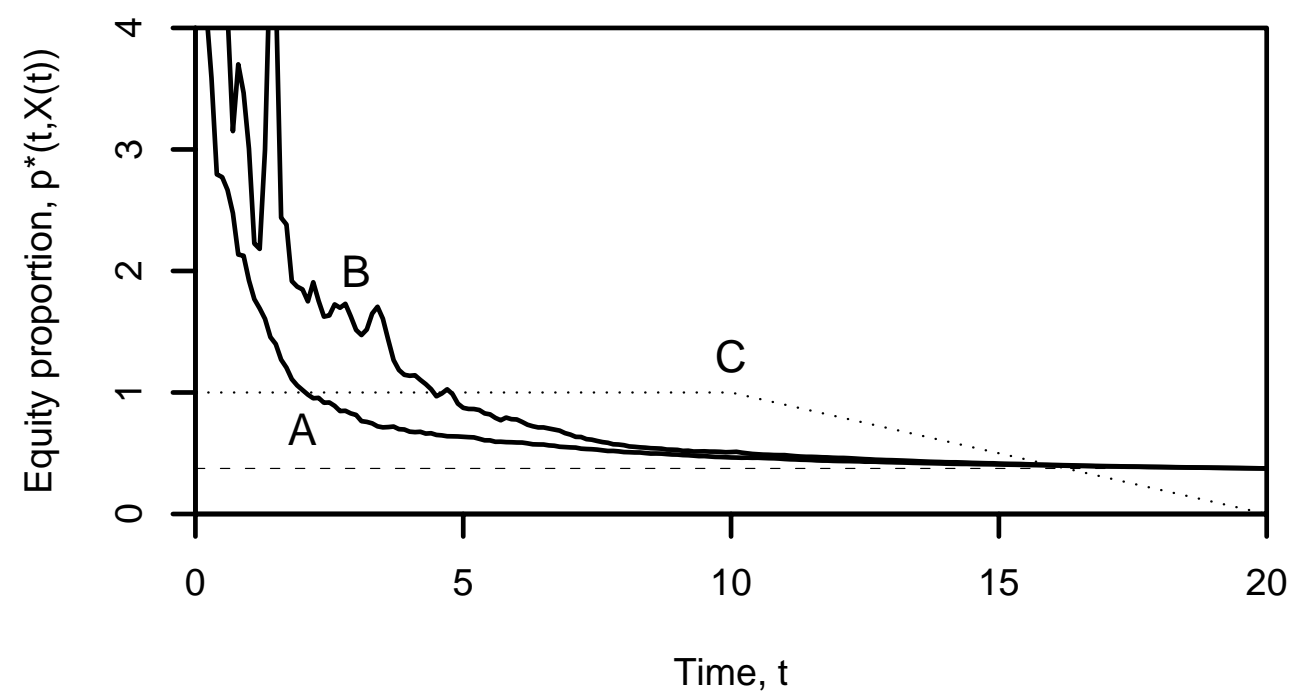

Figure 2: Optimal equity proportions, $p^{*}(t, X(t))$ from two simulated paths (A, B). Parameters as in Figure 1. All simulated paths converge to the dashed line at 0.375 as in Figure 1. Deterministic lifestyle strategy (C: dotted line) is shown for comparison. 


\subsection{Case 4: $\pi>0, \sigma_{Y 0} \neq 0$}

Qualitatively Case 4 is very different from Case 3. In Case 3 we were able to treat future premiums as a quantifiable part of the current assets of the pension fund. In the present case, the market is incomplete so that we are unable to borrow explicitly against future premiums. In particular if $W(t)$ is allowed to become negative then there is a strictly positive probability that $W(T)$ will also be negative (i.e., the plan becomes insolvent). This forces us to exclude asset allocation strategies that will allow wealth to become negative. This means that the optimal strategy for Case 3 is now inadmissible.

We define the general value function $J(t, x, p)=E\left[u\left(X_{p}(T)\right) \mid X(t)=x\right]$, where $X_{p}(t)$ is the path of $X$ given the asset allocation strategy $p=p(t, x)$. Define $\mathcal{P}$ to be the set of all admissible asset allocation strategies, ${ }^{10}$ and define

$$
V(t, x)=\sup _{p \in \mathcal{P}} E\left[u\left(X_{p}(T)\right) \mid X(t)=x\right]=\sup _{p \in \mathcal{P}} J(t, x, p) .
$$

Then $V(t, x)$ satisfies the Hamilton-Jacobi-Bellman equation ${ }^{11}$

$$
\begin{aligned}
V_{t}+\sup _{p \in \mathcal{P}}\left\{\mu_{X}^{p} V_{x}+\frac{1}{2}\left(\sigma_{X}^{p}\right)^{2} V_{x x}\right\} & =0 \\
\text { subject to } V(T, x) & =\frac{1}{\gamma} x^{\gamma} .
\end{aligned}
$$

In this equation $V_{t}, V_{x}$ etc are partial derivatives of $V$ with respect to $t, x$ etc,

$$
\begin{aligned}
\mu_{X}^{p} & =\mu_{X}^{p}(t, x) \\
& =\pi+x\left(-\mu_{Y}+p(t) \sigma_{1}\left(\xi_{1}-\sigma_{Y 1}\right)+\sigma_{Y 0}^{2}+\sigma_{Y 1}^{2}\right) \\
\text { and }\left(\sigma_{X}^{p}\right)^{2} & =\sigma_{X}^{p}(t, x)^{2} \\
& =x^{2}\left(\sigma_{Y 0}^{2}+\left(p(t) \sigma_{1}-\sigma_{Y 1}\right)^{2}\right) .
\end{aligned}
$$

For a given $(t, x)$, we now solve the static supremum problem which results in

$$
p^{*}(t, x) \equiv p^{*}(t, x ; V)=\frac{1}{\sigma_{1}}\left(\sigma_{Y 1}-\frac{V_{x}}{x V_{x x}}\left(\xi_{1}-\sigma_{Y 1}\right)\right) .
$$

The optimal solution for $p$ thus depends on the optimal value function $V(t, x)$,

\footnotetext{
${ }^{10}$ For a discussion of admissible strategies see, for example, Korn (1997).

${ }^{11}$ In the complete market cases ( 1 and 3 ) we could use the alternative martingale approach of Karatzas et al (1987) and Cox \& Huang (1991). This can offer a more direct route to the solution for Cases 1 and 3 but is much less easy to apply in the incomplete market case (Cases 2 and 4). For consistency, then, we focus on the HJB approach.
} 
which is found by solving numerically the PDE

$$
\begin{aligned}
V_{t}+\mu_{X}^{p^{*}} V_{x}+\frac{1}{2}\left(\sigma_{X}^{p^{*}}\right)^{2} V_{x x} & =0 \\
\text { subject to } V(T, x) & =\frac{1}{\gamma} x^{\gamma} \\
\text { where } \mu_{X}^{p^{*}} & =\pi+x\left(-\mu_{Y}+p^{*}(t, x ; V) \sigma_{1}\left(\xi_{1}-\sigma_{Y 1}\right)+\sigma_{Y 0}^{2}+\sigma_{Y 1}^{2}\right) \\
\text { and }\left(\sigma_{X}^{p^{*}}\right)^{2} & =x^{2}\left(\sigma_{Y 0}^{2}+\left\{p^{*}(t, x ; V) \sigma_{1}-\sigma_{Y 1}\right\}^{2}\right) .
\end{aligned}
$$

(Recall that $\mu_{X}^{p^{*}}$ and $\sigma_{X}^{p^{*}}$ are the drift and volatility of $X(t)$ given strategy $p^{*}$.)

\section{Remark 2.4.1}

Equation (2.4.1) shows that the optimal risky portfolio $p^{*}(t, x)$ can be expressed as a combination of two fixed portfolios, $\sigma_{Y 1} / \sigma_{1}$ and $\left(\xi_{1}-\sigma_{Y 1}\right) / \sigma_{1}$, with the balance between the two depending on $t$ and $x$ only through $V(t, x)$.

Figure 3 presents numerical results for $\gamma=-5(R R A=6)$ and the following parameter set:

$$
\mu_{Y}=0, \xi_{1}=0.2, \sigma_{1}=0.2, \sigma_{Y 1}=0.05, \sigma_{Y 0}=0.05, \pi=0.1, T=20 .
$$

In each plot, we give the optimal value function (solid lines) for the terminal utility function $V(t, x)$ for $T-t=10$ and 20 years to maturity. We note from this plot that $V(t, x)$ (for $t<T$ ) has a finite limit as $x \rightarrow 0^{+}$. (In contrast, $V(T, x) \rightarrow-\infty$ as $x \rightarrow 0^{+}$.) This happens because when $X(t)=0$, we will always have $X(T)>0$ due to future premiums and the finite limit for $V(t, x)$ as $x \rightarrow 0^{+}$indicates that $X(T)$ is not concentrated too close to $0 .^{12}$

Figure 3 also plots the suboptimal value function (dashed line) $J(t, x, p)$ when $p=0.375$ for all $(t, x)$ (the limiting value for $p^{*}(t, x)$ as $x \rightarrow \infty$ ). This figure suggests that the differences between the optimal and suboptimal value functions are not too great and diminish as $x$ increases. However, a comparison of expected terminal utilities only tells us that one strategy is better than another and does not allow us to quantify the cost of suboptimality to the plan member. This is an issue that we will come back to in Section 3.4.1.

The corresponding optimal dynamic asset allocation strategies $p^{*}(t, x)$ are plotted in Figure 4 for $T-t=0,10$ and 20 years to maturity. We note that $p^{*}(t, x) \rightarrow \infty$ as $x \rightarrow 0^{+}$(top graph). However, when we look at the amount invested, we observe (particularly from the bottom graph) that $p^{*}(t, x) x$ converges to 0 at the same rate as $\sqrt{x} .{ }^{13}$ This is in clear contrast to Case 3 where $\lim _{x \rightarrow 0} p(t, x) x>0$.

\footnotetext{
${ }^{12}$ Equivalently, the left-hand tail of the distribution of $\log X(T) \mid X(t)=0$ is not too dense.

${ }^{13}$ That is, $\left(p^{*}(t, x) x\right) / \sqrt{x}=p^{*}(t, x) \sqrt{x} \rightarrow c$, for some constant $c \geq 0$, as $x \rightarrow 0$.
} 
The behaviour of $p^{*}(t, x) x$ close to $x$ means that in Case $4 X(t)$ can never become negative (in contrast to Case 3). ${ }^{14}$ It follows that the Case 4 asset allocation strategy is rather more conservative than that of Case 3 when the fund size is low; this is to avoid the risk of bankrupting the fund.

Individual realisations of $p^{*}(t, x)$ against $t$ would look similar to the stochastic lifestyling in Figure 2. However, the square-root convergence property means that the sample paths have slightly lower equity weightings on average. This relationship is consistent with the theory of background risk discussed in Section 2.2 .

\section{$3 \quad$ A more general stochastic model}

\subsection{The structure of the model}

We will now incorporate three extensions to the problem:

- the introduction of a stochastic risk-free nominal rate of interest, $r(t)$;

- the extension of the investment opportunity set to $N$ risky assets rather than 1 ;

- the introduction of the replacement ratio ${ }^{15}$ as an argument in the terminal utility function.

The components of the model are as follows:

- The risk-free rate of interest is a one-factor diffusion process governed by the time-homogeneous SDE

$$
d r(t)=\mu_{r}(r(t)) d t+\sum_{j=1}^{N} \sigma_{r j}(r(t)) d Z_{j}(t)
$$

where the $Z_{i}(t)$ are independent, standard Brownian motions. We define $\sigma_{r}(r)=\left(\sigma_{r 1}(r), \ldots, \sigma_{r N}(r)\right)^{\prime}$. The value of units in the cash fund at $t$ is then $R_{0}(t)=R_{0}(0) \exp \left[\int_{0}^{t} r(s) d s\right]$.

\footnotetext{
${ }^{14}$ In fact, square root convergence is a necessary condition for $X(t)$ to avoid becoming negative (see, for example, Duffie et al., 1997). If $\lim _{x \rightarrow 0} p^{*}(t, x) \sqrt{x}=\infty$ then $X(t)$ could become negative.

${ }^{15}$ The ratio of the initial pension at retirement to the final salary before retirement.
} 


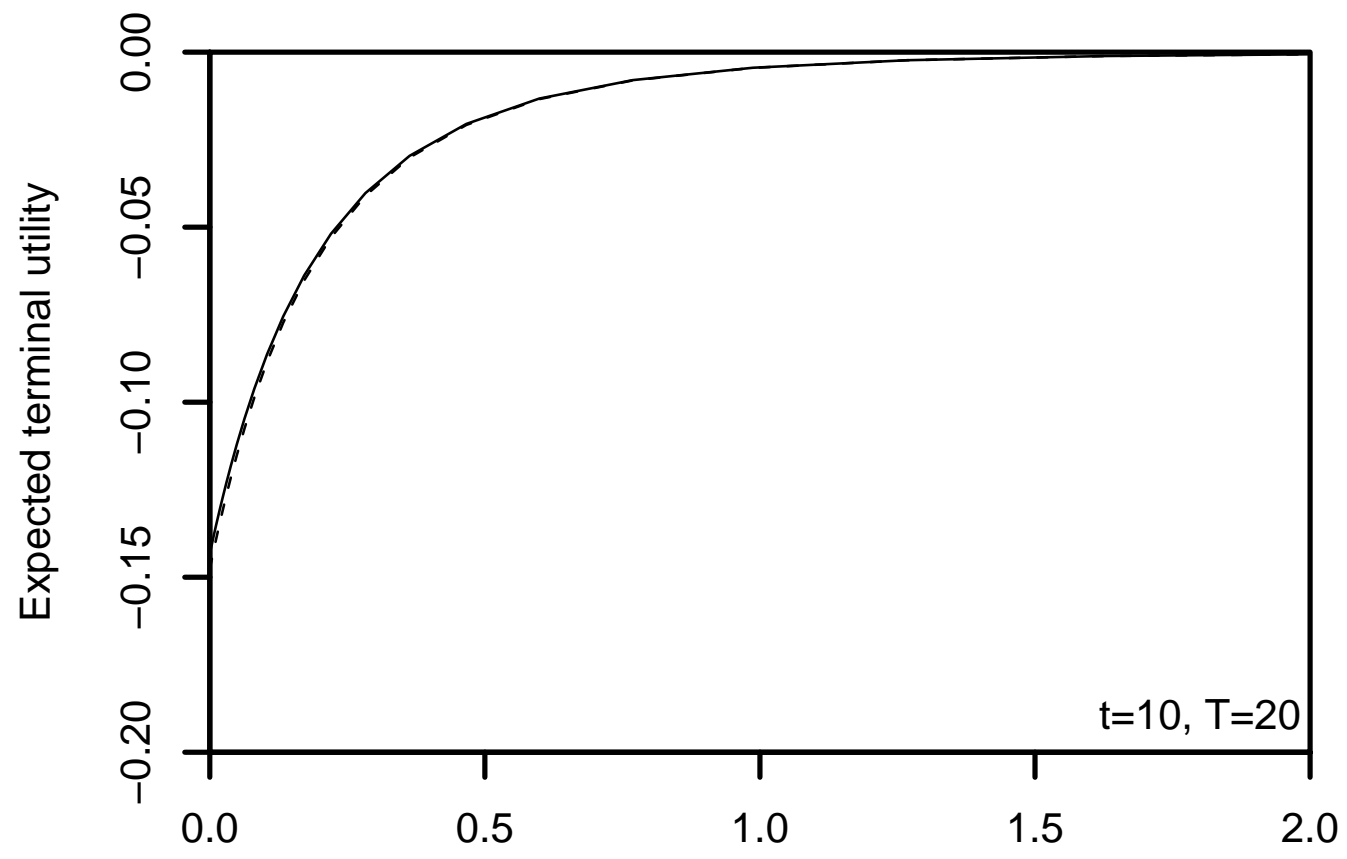

Wealth-income ratio, $X(10)=X$

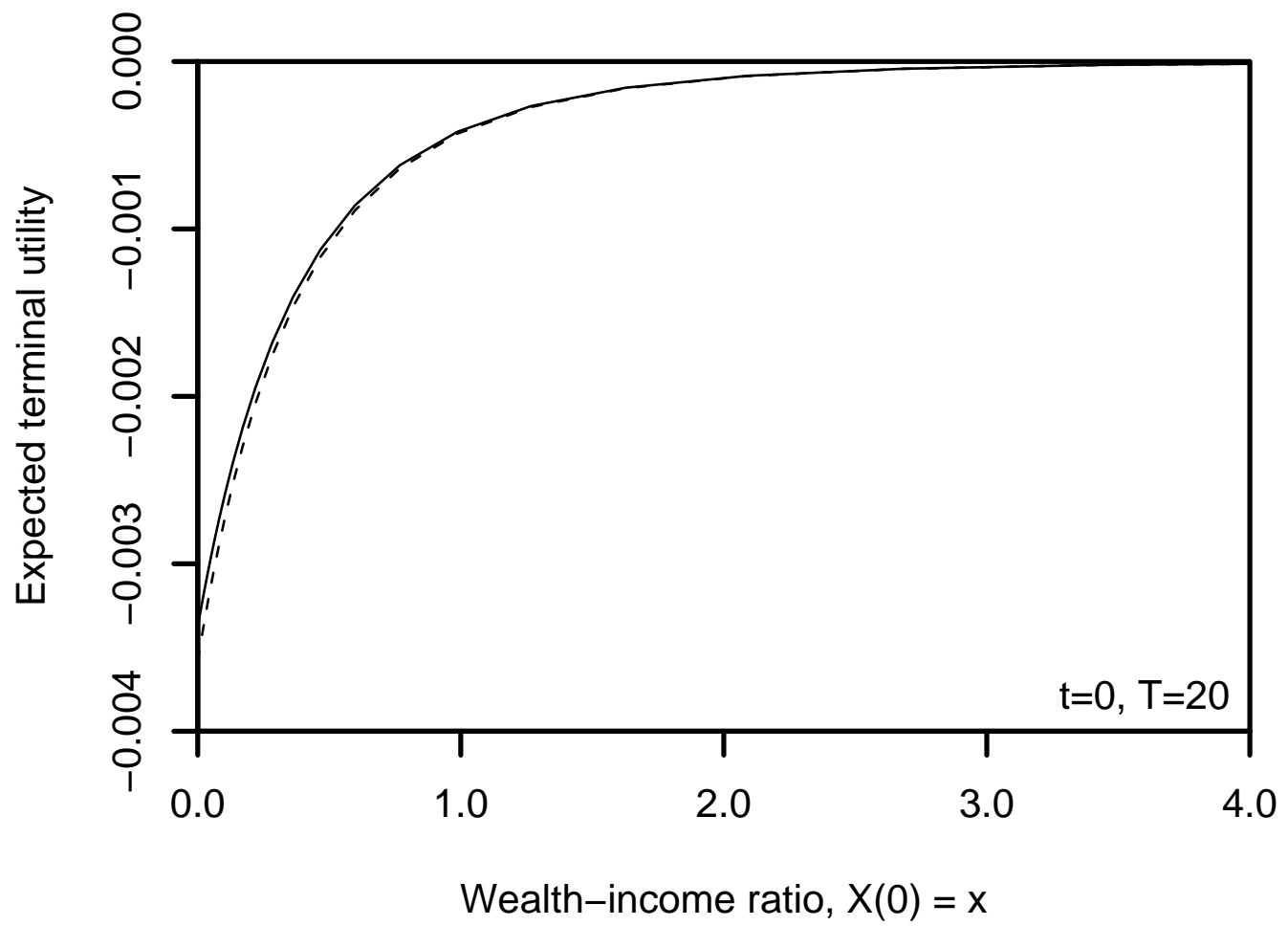

Figure 3: Value functions for 10 and 20 years to maturity when relative risk aversion is $6(\gamma=-5)$ and $\pi=0.1$. Solid lines show the optimal $V(t, x)$, while dashed lines show the suboptimal value function $J(t, x, p)$ where $p=0.375$ for all $t, x$. Both value functions are calculated using the finite difference method. 


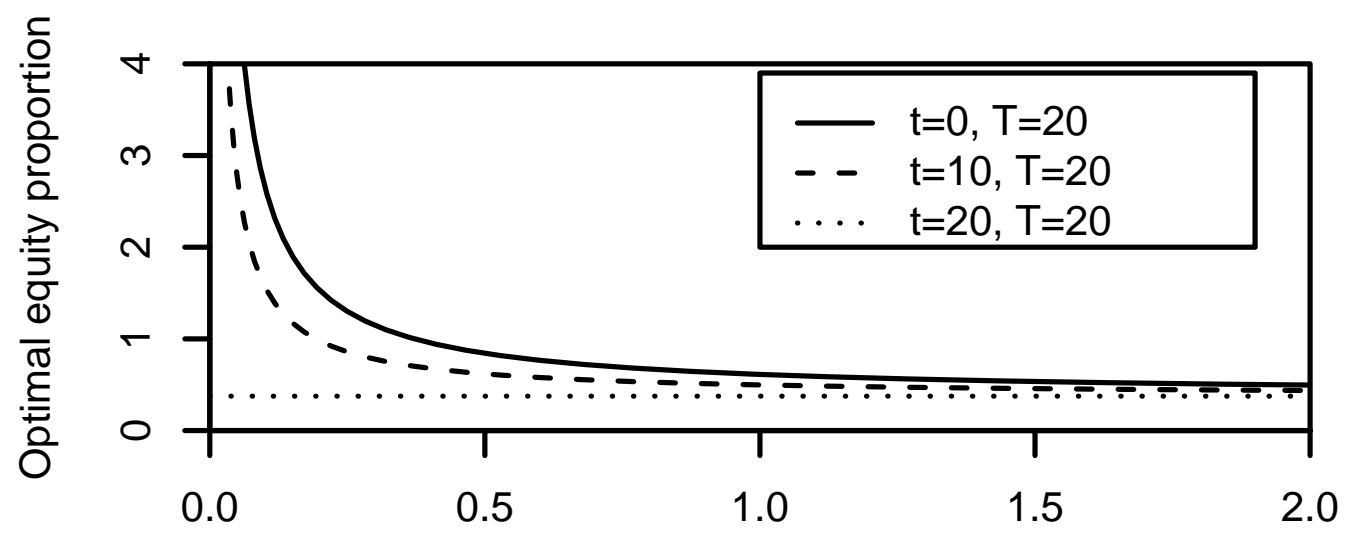

Wealth-income ratio, $X(t)$

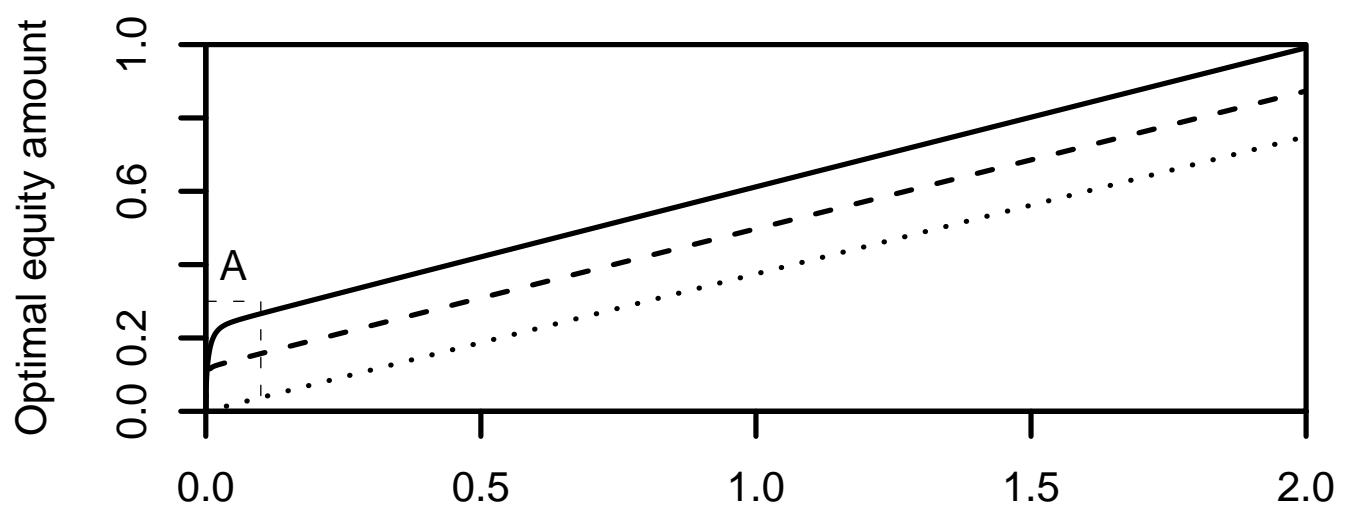

Wealth-income ratio, $X(\mathrm{t})$

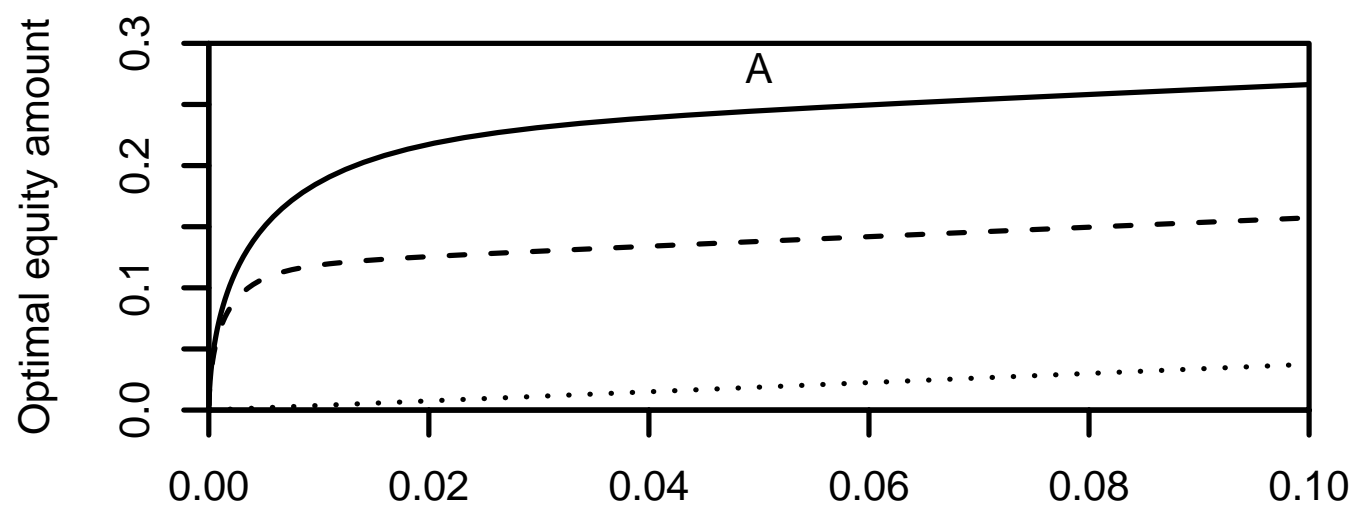

Wealth-income ratio, $X(\mathrm{t})$

Figure 4: Optimal asset-allocation strategies when relative risk aversion is $6(\gamma=$ -5 ), $\pi=0.1$ and $T=20$. Top: equity proportions $p^{*}(t, x)$ for $t=0$ (solid line), $t=10$ (dashed) and $t=20$ (dotted). Middle: amounts invested in equities, $p^{*}(t, x) x$, for $t=0$ (solid line), $t=10$ (dashed) and $t=20$ (dotted). Bottom: box A in the middle graph magnified. 
- There are $N$ risky assets. Let $R_{i}(t)$ be the total return ${ }^{16}$ on an investment solely in asset $i$ with

$$
d R_{i}(t)=R_{i}(t)\left[\left(r(t)+\sum_{j=1}^{N} \sigma_{i j} \xi_{j}\right) d t+\sum_{j=1}^{N} \sigma_{i j} d Z_{j}(t)\right]
$$

for $i=1, \ldots, N$. The volatility matrix $C=\left(\sigma_{i j}\right)_{i, j=1}^{N}$ is assumed to be constant as are the market prices of risk, the $\xi=\left(\xi_{1}, \ldots, \xi_{N}\right)^{\prime}$. The risk premium on asset $i$ is $\sum_{j=1}^{N} \sigma_{i j} \xi_{j}$.

- The plan member's salary, $Y(t)$, evolves according to the SDE

$$
d Y(t)=Y(t)\left[\left(r(t)+\mu_{Y}(t)\right) d t+\sigma_{Y 0} d Z_{0}(t)+\sum_{j=1}^{N} \sigma_{Y j} d Z_{j}(t)\right]
$$

where $\mu_{Y}(t)$ is a deterministic function of time, the $\sigma_{Y j}$ 's are constants and $Z_{0}(t)$ is a standard Brownian motion, independent of $Z_{1}(t), \ldots, Z_{N}(t)$. We define the vector $\sigma_{Y}=\left(\sigma_{Y 1}, \ldots, \sigma_{Y N}\right)^{\prime}$.

- The value of the plan member's pension fund (pension wealth) is denoted by $W(t)$ and has the SDE

$$
d W(t)=W(t)\left[\left(r(t)+p(t)^{\prime} C \xi\right) d t+p(t)^{\prime} C d Z(t)\right]+\pi Y(t) d t
$$

where $Z(t)=\left(Z_{1}(t), \ldots, Z_{N}(t)\right)^{\prime}$ and $p(t)=\left(p_{1}(t), \ldots, p_{N}(t)\right)^{\prime}$ are the proportions of the fund invested in the various risky assets.

In this problem, the control variable is $p(t)$, which we will initially take to be unconstrained. The set $\mathcal{P}$ will be used to denote the set of all admissible controls, $p(t)$.

- At the time of retirement at age $\zeta$, the fund is used to purchase a pension at the prevailing market rate for life annuities, $a(T, r(T))$. For example, for a level annuity of 1 unit per annum payable continuously we have (for a general retirement date $t$ )

$$
a(t, r(t))=\int_{0}^{\infty} b(t, t+s ; r(t)) \phi_{\zeta}(t, s) d s
$$

Here

$-b(t, \tau ; r)$ is the price at time $t$ of a zero-coupon bond maturing at time $\tau$ when the risk-free rate of interest at time $t$ is $r$.

\footnotetext{
${ }^{16}$ That is, the value of a single premium investment in asset $i$ with reinvestment of dividend income.
} 
- $\phi_{\zeta}(t, s)$ is the probability of survival from time $t$ to $t+s$ for a life aged $\zeta$ at time $t$. We assume that $\phi_{\zeta}(t, s)$ is known at time 0 .

The rate per annum of the continuously-paid pension purchased at $T$ is

$$
P(T)=\frac{W(T)}{a(T, r(T))} .
$$

- We assume the plan member's terminal utility depends on both his final salary and his pension wealth at retirement. We will focus on two special cases: (a) the ratio of terminal pension wealth to final salary, $X(T)=$ $W(T) / Y(T)$, and (b) the replacement ratio, $H(T)=P(T) / Y(T)=$ $X(T) / a(T, r(T))$. Thus terminal utility will be of the form

$$
u(X(T), r(T)) \equiv u(X(T)) \quad \text { or } \quad u(X(T) / a(T, r(T))) .
$$

If utility is some function of $P(T) / Y(T)^{17}$ the effect of habit formation is more apparent (in the sense proposed by Ryder and Heal, 1973, Sundaresan, 1989, and Constantinides, 1990), since we are comparing consumption after retirement with the rate of consumption just before retirement.

Expected terminal utility is then given by

$$
J(t, x, r ; p)=E\left[u\left(X_{p}(T), r(T)\right) \mid X(t)=x, r(t)=r\right]
$$

where $X_{p}(t)$ is the path of $X(t)$ given the strategy $p$.

Our aims are twofold. The first is to determine the plan member's optimal expected terminal utility, that is, to find

$$
V(t, x, r)=\sup _{p \in \mathcal{P}} J(t, x, r ; p)
$$

and to determine the strategy $p$ that attains this maximum. In addition, we wish to evaluate the performance of a variety of popular asset allocation strategies relative to this theoretical benchmark.

Given the wealth to salary ratio, $X(t)=W(t) / Y(t)$, a straightforward application of the product rule gives us the SDE

$$
\begin{array}{r}
d X(t)=X(t)\left[\left(-\mu_{Y}(t)+p(t)^{\prime} C\left(\xi-\sigma_{Y}\right)+\sigma_{Y 0}^{2}+\sigma_{Y}^{\prime} \sigma_{Y}\right) d t\right. \\
\left.-\sigma_{Y 0} d Z_{0}(t)+\left(p(t)^{\prime} C-\sigma_{Y}^{\prime}\right) d Z(t)\right]+\pi d t
\end{array}
$$

\footnotetext{
${ }^{17}$ More precisely, we could look at $P(T) /(1-\pi) Y(T)$ since $(1-\pi) Y(T)$ and $P(T)$ are the consumption rates just before and just after retirement. However, it is not necessary for us to include explicitly the $(1-\pi)$ factor. First, $\pi$ is an exogenously specified parameter. Second, since we are using power utility the $(1-\pi)$ factor results in a constant multiplier in the utility that has no impact on the optimisation problem. For these reasons we prefer to leave out the $(1-\pi)$.
} 


\subsection{The Hamilton-Jacobi-Bellman equation}

Our approach to solving the optimisation problem (equation 3.1.2) is to use the Hamilton-Jacobi-Bellman (HJB) equation. The HJB equation for this problem is (see, for example, Merton, 1969, 1971, 1990, Korn, 1997, Øksendal, 1998, or Björk, 1998)

$$
V_{t}+\sup _{p \in \mathcal{P}}\left\{\mathcal{A}^{p} V\right\}=0
$$

where

$$
\begin{aligned}
\mathcal{A}^{p} & =\mu_{r}(r) \frac{\partial}{\partial r}+\mu_{X}^{p} \frac{\partial}{\partial x}+\frac{1}{2} \nu_{r r} \frac{\partial^{2}}{\partial r^{2}}+\nu_{r x}^{p} \frac{\partial^{2}}{\partial r \partial x}+\frac{1}{2} \nu_{x x}^{p} \frac{\partial^{2}}{\partial x^{2}} \\
\mu_{X}^{p} & =x\left(-\tilde{\mu}_{Y}(t)+p^{\prime} C\left(\xi-\sigma_{Y}\right)\right)+\pi \\
\tilde{\mu}_{Y}(t) & =\mu_{Y}(t)-\sigma_{Y 0}^{2}-\sigma_{Y}^{\prime} \sigma_{Y} \\
\nu_{r r} & =\sigma_{r}(r)^{\prime} \sigma_{r}(r) \\
\nu_{r x}^{p} & =\left(p^{\prime} C-\sigma_{Y}^{\prime}\right) \sigma_{r}(r) x \\
\text { and } \nu_{x x}^{p} & =\left(\sigma_{Y 0}^{2}+\left(p^{\prime} C-\sigma_{Y}^{\prime}\right)\left(C^{\prime} p-\sigma_{Y}\right)\right) x^{2} .
\end{aligned}
$$

If we follow the usual steps (see, for example, Björk, 1998) we find that the optimal asset allocation strategy takes the form

$$
p^{*}(t, x, r ; V)=C^{\prime-1}\left(\sigma_{Y}-\left(\xi-\sigma_{Y}\right) \frac{V_{x}}{x V_{x x}}-\sigma_{r}(r) \frac{V_{x r}}{x V_{x x}}\right) .
$$

If we now insert this expression for $p^{*}(t, x, r ; V)$ into equation (3.2.1) and simplify we get the PDE for $V(t, x, r)$

$$
\begin{aligned}
V_{t}+\mu_{r}(r) & V_{r}+\left(\pi-\tilde{\mu}_{Y}(t) x+\sigma_{Y}^{\prime}\left(\xi-\sigma_{Y}\right) x\right) V_{x}+\frac{1}{2} \sigma_{r}(r)^{\prime} \sigma_{r}(r) V_{r r}+\frac{1}{2} \sigma_{Y 0}^{2} x^{2} V_{x x} \\
- & \frac{1}{2}\left(\xi-\sigma_{Y}\right)^{\prime}\left(\xi-\sigma_{Y}\right) \frac{V_{x}^{2}}{V_{x x}}-\left(\xi-\sigma_{Y}\right)^{\prime} \sigma_{r}(r) \frac{V_{x} V_{x r}}{V_{x x}}-\frac{1}{2} \sigma_{r}(r)^{\prime} \sigma_{r}(r) \frac{V_{x r}^{2}}{V_{x x}}=0 .
\end{aligned}
$$

Before we go on to look at the solution of the PDE (3.2.3) we will make some observations about the composition of the optimal portfolio $p^{*}(t, x, r ; V)$ in equation (3.2.2).

\section{Theorem 3.2.1 [Three-fund theorem]}

The optimal asset mix, $p^{*}(t, x, r ; V)$ (equation 3.2.2), at any given time, consists 
of investments in three efficient mutual funds as follows:

$$
\begin{aligned}
& p^{*}(t, x, r ; V)=\theta_{A} p_{A}+\theta_{B} p_{B}+\theta_{C} p_{C} \\
& \text { where } \theta_{A}=\theta_{A}(t, x, r)=1-\frac{V_{x r}-d_{a}(r) V_{x}}{d_{a}(r) x V_{x x}} \\
& \theta_{B}=\theta_{B}(t, x, r)=\frac{V_{x r}}{d_{a}(r) x V_{x x}} \\
& \text { and } \theta_{C}=\theta_{C}(t, x, r)=1-\theta_{A}(t, x, r)-\theta_{B}(t, x, r)=-\frac{V_{x}}{x V_{x x}} \\
& \text { with } p_{A}=C^{\prime-1} \sigma_{Y} \\
& p_{B}=C^{\prime-1}\left(\sigma_{Y}-d_{a}(r) \sigma_{r}(r)\right) \\
& \text { and } p_{C}=C^{-1} \xi \text {. }
\end{aligned}
$$

\section{Proof: See Appendix A.}

We interpret (3.2.4) and (3.2.5) as follows. The optimal weight in risky assets is equivalent to investing in three efficient mutual funds denoted A, B and C. The three mutual funds can be interpreted as follows:

A: Fund A is the minimum-risk portfolio measured relative to the salary numeraire, $Y(t)$, and its purpose is to hedge salary risk. Asset proportions are given by the vector $p_{A}$ (with $p_{A 0}=1-\sum_{i=1}^{N} p_{A i}$ ). Since this fund will be dominated by cash (and contain 100\% cash if salary growth and asset returns are uncorrelated, but also contain other assets if salary growth is correlated with their returns), we will refer to Fund A for convenience as the 'cash' fund.

B: Fund B is the minimum-risk portfolio measured relative to $Y(t) / a(t, r(t))$ and its purpose is to hedge annuity risk. Asset proportions are given by the vector $p_{B}$ (with $p_{B 0}=1-\sum_{i=1}^{N} p_{B i}$ ). Since this fund will be dominated by bonds whose returns are highly correlated with annuity yields, we will refer to Fund B as the 'bond' fund.

$\mathrm{C}$ : Fund $\mathrm{C}$ is a risky portfolio which is efficient when measured relative to both $Y(t)$ and $Y(t) / a(t, r(t))$. Asset proportions are given by the vector $p_{C}$ (with $\left.p_{C 0}=1-\sum_{i=1}^{N} p_{C i}\right)$. The fund will be dominated by equities and so we will refer to it as the 'equity' fund: its purpose is to satisfy the risk appetite of the plan member.

Mutual funds A and C (equation 3.2.5) maintain constant proportions in each of the $N+1$ assets. The equivalent proportions in fund $\mathrm{B}$ will vary over time, but 
only in response to changes in $r(t)$ rather than, separately, to changes in $t$ or $X(t)$. However, the proportions of the overall pension fund invested in each of the three mutual funds (that is, $\theta_{A}(t, x, r), \theta_{B}(t, x, r), \theta_{C}(t, x, r)$ ) depend on all of $t, X(t)$ and $r(t)$, according to equations (3.2.5). As in Section 2 the stochastic nature of the paths of $\theta_{A}(t, x, r), \theta_{B}(t, x, r)$ and $\theta_{C}(t, x, r)$ over time means that the optimal asset allocation strategy can usefully be described as stochastic lifestyling.

\section{Remark 3.2.2}

The term $\theta_{C}$ is equal to the reciprocal of the degree of relative risk aversion.

It follows that, since relative risk aversion is positive (but possibly dependent upon $t$ and $x$ ), then the investment in portfolio $C$ is necessarily positive. In addition, if relative risk aversion is constant, it will be optimal to invest a constant proportion in the risky portfolio $C^{-1} \xi$ over time.

\section{Remark 3.2.3}

The three portfolios, $p_{A}, p_{B}$ and $p_{C}$, do not depend upon the level of non-hedgeable salary risk, $\sigma_{Y 0}$. However, the precise mix ${ }^{18}$ will depend upon $\sigma_{Y 0}$ through its effect on $V(t, x, r)$.

Provided that all members have the same values for $\sigma_{Y}$, the same three funds can be used for all plan members no matter what their idiosyncratic risk $\left(\sigma_{Y 0}\right)$, age, wealth or attitude to risk. This has the important practical consequence that pension providers can use these three general funds to satisfy the needs of many plan members instead of having to provide tailor-made portfolios for each individual.

\section{Corollary 3.2.4}

Suppose that $V(T, x, r)=K(x / a(T, r))$, that is, the terminal utility is a function of the pension as a proportion of final salary (replacement ratio) achieved at time $T$. Then $\theta_{A}(T, x, r)=0$ for all $x, r$.

\section{Proof:}

\footnotetext{
${ }^{18}$ We will see how this mix varies stochastically in the numerical example later in this section.
} 
At $t=T$, we find that:

$$
\begin{aligned}
V_{x} & =\frac{1}{a(T, r)} K^{\prime}(x / a(T, r)) \\
V_{x r} & =\frac{d_{a}(r)}{a(T, r)} K^{\prime}(x / a(T, r))+\frac{x d_{a}(r)}{a(T, r)^{2}} K^{\prime \prime}(x / a(T, r)) \\
V_{x x} & =\frac{1}{a(T, r)^{2}} K^{\prime \prime}(x / a(T, r)) .
\end{aligned}
$$

It is then straightforward to confirm that $\theta_{A}(T, x, r)=0$.

This result shows what happens to the asset allocation when the plan member is concerned about receiving a pension at retirement, rather than a cash lump sum. Although part of the pension wealth will generally be invested in fund A prior to retirement, as the retirement date approaches, the weight in fund $\mathrm{A}$ is reduced to zero. The exception to this result occurs if the pension plan is funding for a cash lump sum at retirement rather than a pension. In this case $d_{a}(r)=0$ for all $r$ and portfolios A and B are identical.

\section{Conjecture 3.2.5}

As $T-t$ tends to infinity $\theta_{B}(t, x, r)$ tends to zero.

We make this conjecture on the following basis. The further we are from retirement the less able are we to predict what interest rates will be at the time of retirement. This means that $V_{r}$ and $V_{x r}$ are likely to tend to 0 as $T-t$ increases. For a specific example where the conjecture is true, see Section 3.4.1 and Equation (3.4.7).

\subsubsection{Boundary conditions for the PDE}

So far we have derived general results which do not depend upon a specific form for $u(x, r)$. However, if we wish to obtain further results, we need to be more specific about the terminal utility, $u(x, r)$. This will give us the boundary condition for the PDE (equation 3.2.3: $V(T, x, r)=u(x, r)$ ). In the sections that follow, we we will narrow our analysis to terminal utilities which are power utilities in $x$, while keeping a more general functional form in $r$ : that is,

$$
u(x, r)=\frac{1}{\gamma} g(T, r)^{1-\gamma} x^{\gamma}
$$

for some general function $g(T, r)$. A full power utility function will be employed in the numerical example in Section 3.4.1.

We are now in a position to discuss two of the four cases analysed previously in Section 2. Case $2\left(\pi=0, \sigma_{Y 0} \neq 0\right)$ and Case $4\left(\pi>0, \sigma_{Y 0} \neq 0\right)$ involve a type 
of computational analysis that is sufficiently different and sufficiently extended to justify a separate paper. ${ }^{19}$

\subsection{Case 1: $\pi=0, \sigma_{Y 0}=0$}

Let us first consider the solution for (3.2.3) for the single-premium Case 1 where $\pi=0$ and $\sigma_{Y 0}=0$. While this case is not particularly interesting in itself, it leads us directly to the solution for Case 3 where $\pi>0$ and $\sigma_{Y 0}=0$.

\section{Theorem 3.3.1}

$V(t, x, r)$ is of the form $\gamma^{-1} g(t, r)^{1-\gamma} x^{\gamma}$ where $g(t, r)$ satisfies the PDE

$$
\begin{aligned}
g_{t}+\frac{1}{2} \sigma_{r}(r)^{\prime} \sigma_{r}(r) g_{r r}+\left(\mu_{r}(r)+\frac{\gamma}{(1-\gamma)}\left(\xi-\sigma_{Y}\right)^{\prime} \sigma_{r}(r)\right) g_{r} \\
\quad+\frac{\gamma}{(1-\gamma)}\left[-\mu_{Y}(t)+\sigma_{Y}^{\prime} \xi-\frac{1}{2(\gamma-1)}\left(\xi-\sigma_{Y}\right)^{\prime}\left(\xi-\sigma_{Y}\right)\right] g=0 .
\end{aligned}
$$

The boundary condition for $g(T, r)$ is defined as $\left\{\gamma V(T, x, r) x^{-\gamma}\right\}^{1 /(1-\gamma)}$ or $\left\{\gamma u(x, r) x^{-\gamma}\right\}^{1 /(1-\gamma)}$.

Proof: See Appendix.

\section{Corollary 3.3.2}

By the Feynman-Kac formula (see, for example, Björk, 1998) there exists a probability measure ${ }^{20} Q(\gamma)$ such that

$$
g(t, r(t))=E_{Q(\gamma)}\left[g(T, \tilde{r}(T)) D(t, T) \mid \mathcal{F}_{t}\right]
$$

where $\tilde{r}(s)$ is governed by the SDE

$$
\begin{aligned}
d \tilde{r}(s) & =\tilde{\mu}_{r}(\tilde{r}(s)) d s+\left|\sigma_{r}(\tilde{r}(s))\right| d \tilde{Z}(s) \\
\tilde{\mu}_{r}(r) & =\mu_{r}(r)+\frac{\gamma}{1-\gamma}\left(\xi-\sigma_{Y}\right)^{\prime} \sigma_{r}(r) \\
\tilde{r}(t) & =r(t)
\end{aligned}
$$

\footnotetext{
${ }^{19}$ However, we conjecture that the outcomes would be a combination of what we have already observed in both the deterministic and stochastic $r(t)$ models.

${ }^{20}$ The measure $Q(\gamma)$ is (like the risk-neutral measure in derivative pricing) an artificial probability measure which provides us with a convenient computational tool. It does not imply that investors with different levels of risk aversion use different probabilities.
} 
where $\left|\sigma_{r}(\tilde{r}(s))\right|$ is the modulus of $\sigma_{r}(\tilde{r}(s)), \tilde{Z}(s)$ is a standard, one-dimensional Brownian motion under the artificial measure $Q(\gamma)$,

$$
\begin{aligned}
D(t, T) & =\exp \left[\frac{\gamma}{1-\gamma} \int_{t}^{T} \theta(s) d s\right] \\
\theta(s) & =-\mu_{Y}(s)+\sigma_{Y}^{\prime} \xi-\frac{1}{2(\gamma-1)}\left(\xi-\sigma_{Y}\right)^{\prime}\left(\xi-\sigma_{Y}\right) \\
\Rightarrow D(t, T) & =\exp \left[\frac{\gamma}{1-\gamma}\left\{-M_{Y}(t, T)+\psi(\gamma)(T-t)\right\}\right] \\
M_{Y}(t, T) & =\int_{t}^{T} \mu_{Y}(s) d s \\
\text { and } \psi(\gamma) & =\sigma_{Y}^{\prime} \xi-\frac{1}{2(\gamma-1)}\left(\xi-\sigma_{Y}\right)^{\prime}\left(\xi-\sigma_{Y}\right) .
\end{aligned}
$$

Consider the optimal asset mix. The general form for $V(t, x, r)$ reveals immediately that the proportion of the fund invested in portfolio $p_{C}$ is constant: that is, $\theta_{C}(t, x, r)=1 /(1-\gamma)$ for all $(t, x, r)$. The proportions $\theta_{A}$ and $\theta_{B}$ invested in $p_{A}$ and $p_{B}$, respectively, will depend upon $t$ and $r$ but not upon $x$. In particular, we note that since $E_{Q(\gamma)}\left[g(T, \tilde{r}(T)) \mid \mathcal{F}_{t}\right]$ depends upon the particular model chosen for $r(t)$, so will $\theta_{A}(t, x, r)$ and $\theta_{B}(t, x, r)$.

Suppose also that $r(t)$ is stationary and ergodic under $Q(\gamma)$ and that $E_{Q(\gamma)}\left[g(T, \tilde{r}(T)) \mid \mathcal{F}_{t}\right] \rightarrow$ constant and is finite as $T-t \rightarrow \infty$. Then, as $T-t \rightarrow$ $\infty, g(T, r(t)) / D(t, T) \rightarrow$ constant for all $r(t)$. It follows that the proportion, $\theta_{B}(t, x, r)$, invested in $p_{B}$ tends to 0 as $T-t \rightarrow \infty$ (Conjecture 3.2.5).

On the other hand, if the plan is funding for a cash lump sum at $T$, rather than a pension, then $g(T, r) \equiv 1$ and $\theta_{B}(t, x, r) \equiv 0$ for all $(t, x, r)$ (see equation (3.2.5)).

\subsection{Case 3: $\pi>0, \sigma_{Y 0}=0$}

Since salaries are hedgeable, we can attach a unique price to future pension contributions. Let $Q$ be the risk-neutral pricing measure under which the $N$ risky assets have the dynamics

$$
d R_{i}(t)=R_{i}(t)\left[r(t) d t+\sum_{j=1}^{N} \sigma_{i j} d \tilde{Z}_{j}(t)\right]
$$


and where the $\tilde{Z}_{j}(t)$ are independent standard $Q$-Brownian motions. Also we have under $Q$

$$
\begin{gathered}
d Y(t)=Y(t)\left[\left(r(t)+\mu_{Y}(t)-\sum_{j=1}^{N} \sigma_{Y j} \xi_{j}\right) d t+\sum_{j=1}^{N} \sigma_{Y j} d \tilde{Z}_{j}(t)\right] \\
\Rightarrow Y(\tau)=Y(t) \exp \left\{\int_{t}^{\tau}\left(r(s)+\mu_{Y}(s)\right) d s-\sum_{j=1}^{N} \sigma_{Y j}\left(\xi_{j}+\frac{1}{2} \sigma_{Y j}\right)(\tau-t)\right. \\
\left.+\sum_{j=1}^{N} \sigma_{Y j}\left(\tilde{Z}_{j}(\tau)-\tilde{Z}_{j}(t)\right)\right\} .
\end{gathered}
$$

The market value at time $t$ for future premiums payable between $t$ and $T$ is then

$E_{Q}\left[\int_{t}^{T} \exp \left\{-\int_{t}^{\tau} r(s) d s\right\} \pi Y(\tau) d \tau \mid \mathcal{F}_{t}\right]$

$=\pi E_{Q}\left[\int_{t}^{T} Y(t) \exp \left\{\int_{t}^{\tau} \mu_{Y}(s) d s-\sigma_{Y}^{\prime} \xi(\tau-t)-\frac{1}{2}\left|\sigma_{Y}\right|^{2}(\tau-t)+\sigma_{Y}^{\prime}(\tilde{Z}(\tau)-\tilde{Z}(t))\right\} d \tau \mid \mathcal{F}_{t}\right]$

$=\pi Y(t) \int_{t}^{T} \exp \left\{M_{Y}(t, \tau)-\sigma_{Y}^{\prime} \xi(\tau-t)\right\} d \tau \quad$ (by 3.3.2)

$=\pi Y(t) f(t), \quad$ say.

\section{Theorem 3.4.1}

$V(t, x, r)$ is of the form $\gamma^{-1} g(t, r)^{1-\gamma}(x+\pi f(t))^{\gamma}$ where $g(t, r)$ satisfies the PDE (3.3.1) with boundary condition $g(T, r)=\gamma V(T, x, r) x^{-\gamma}$.

Proof: We only need to note that the optimal strategy is composed of two parts.

At time $t$ :

A: hold $-\pi Y(t) f(t)$ in the replicating portfolio which will be paid off exactly by future premiums;

B: invest the surplus $\tilde{W}(t)=W(t)+\pi Y(t) f(t)$ in portfolios $p_{A}, p_{B}$ and $p_{C}$ in the same proportions $\theta_{A}, \theta_{B}$ and $\theta_{C}$ as in Case 1 where $\pi=0$ and $\sigma_{Y 0}=0$.

This will produce the same expected terminal utility as the single premium Case

1. Any other strategy will generate a lower value. 


\subsubsection{A numerical example using the Vasicek model}

Let us now illustrate how the optimal asset allocation strategy varies with $(t, x, r)$ for a specific example. In this example we will assume that plan members contribute to their pension plan at the rate of $10 \%$ of salary per annum: that is, $\pi=0.1$.

We will now use the Vasicek (1977) model for $r(t)$, which will allow us to derive tractable results. Further, bearing in mind the three-fund theorem above, we can restrict the asset allocation to three mutual funds, a cash fund, a bond fund and an equity fund. Thus we will take

$$
d r(t)=\alpha_{r}\left(\mu_{r}-r(t)\right) d t+\sigma_{r 1} d Z_{1}(t)
$$

with $\sigma_{r 2}=0$. The parameter values in our model will be

$$
\begin{aligned}
& \alpha_{r}=0.25, \quad \mu_{r}=0.06, \quad \sigma_{r 1}=-0.02 \\
& C=\left(\begin{array}{cc}
0.1 & 0 \\
0.1 & 0.2
\end{array}\right), \quad \xi=\left(\begin{array}{l}
0.2 \\
0.3
\end{array}\right), \quad \sigma_{Y}=\left(\begin{array}{c}
0.02 \\
0.02
\end{array}\right) .
\end{aligned}
$$

From the structure of $C$ and $\sigma_{r}$ we see that $R_{1}(t)$ is the bond fund and that $R_{2}(t)$ is the equity fund.

For simplicity, we will assume that $a(t, r(t))$ is of the form $\exp \left[d_{0}-d_{1} r(t)\right]$. This will keep things tractable without seriously altering the qualitative observations in our example. We will assume that $d_{0}=3$ and $d_{1}=3.5$ (which implies that $a(t, r(t))$ behaves like a zero-coupon bond with 8.318 years to maturity).

It follows that

$$
\begin{aligned}
& p_{A}=C^{\prime-1} \sigma_{Y}=\left(\begin{array}{c}
0.1 \\
0.1
\end{array}\right), \quad p_{C}=C^{\prime-1} \xi=\left(\begin{array}{c}
0.5 \\
1.5
\end{array}\right), \\
& \text { and } \quad p_{B}=C^{\prime-1}\left(\sigma_{Y}-d_{1} \sigma_{r}\right)=\left(\begin{array}{c}
0.8 \\
0.1
\end{array}\right) .
\end{aligned}
$$

Now, under the artificial measure $Q(\gamma)$

$$
\begin{aligned}
d \tilde{r}(s) & =\tilde{\mu}_{r}(\tilde{r}(s)) d s+\sigma_{r 1} d \tilde{Z}(s) \\
\text { where } \tilde{\mu}_{r}(r) & =\alpha_{r}\left(\tilde{\mu}_{r}-r\right) \\
\text { and } \tilde{\mu}_{r} & =\mu_{r}+\frac{\gamma\left(\xi_{1}-\sigma_{Y 1}\right) \sigma_{r 1}}{(1-\gamma) \alpha_{r}}=0.06-0.0144 \frac{\gamma}{1-\gamma} .
\end{aligned}
$$

We will now take the terminal utility function

$$
\begin{aligned}
u(x, r)=V(T, x, r) & =\frac{1}{\gamma}\left(\frac{x}{a(T, r)}\right)^{\gamma}=\frac{1}{\gamma} e^{-\gamma d_{0}+\gamma d_{1} r} x^{\gamma} \\
\Rightarrow g(T, r) & =\exp \left[\frac{\gamma}{1-\gamma}\left(-d_{0}+d_{1} r\right)\right] .
\end{aligned}
$$


We will make the final parameter choices as follows:

$$
\gamma=-5, \quad \mu_{Y} \equiv 0
$$

Having defined $g(T, r)$ we can now use Corollary 3.3.2 to derive a functional form for $g(t, r)$. The various quantities defined in equation (3.3.2) are $\psi(\gamma)=0.1092333$ (as required for $D(t, T)$ ), $\tilde{\mu}_{r}=0.072$, and $M_{Y}(t, T) \equiv 0$. Under $Q(\gamma), \tilde{r}(T)$, given $r(t)$, is normally distributed with

$$
\begin{aligned}
E_{Q(\gamma)}[\tilde{r}(T) \mid r(t)] & =\tilde{\mu}_{r}+\left(r(t)-\tilde{\mu}_{r}\right) e^{-\alpha_{r}(T-t)} \\
\text { and } \operatorname{Var}_{Q(\gamma)}[\tilde{r}(T) \mid r(t)] & =\sigma_{r 1}^{2} \frac{\left(1-e^{-2 \alpha_{r}(T-t)}\right)}{2 \alpha_{r}} \\
\Rightarrow E_{Q(\gamma)}[g(T, \tilde{r}(T)) \mid r(t)] & =\exp \left[\frac{A(\gamma, T-t)+B(\gamma, T-t) r(t)}{1-\gamma}\right] \\
\text { where } A(\gamma, \tau) & =-\gamma d_{0}+\gamma d_{1} \tilde{\mu}_{r}\left(1-e^{-\alpha_{r} \tau}\right)+\frac{1}{2} \frac{\gamma^{2} d_{1}^{2} \sigma_{r 1}^{2}}{(1-\gamma)} \frac{\left(1-e^{-2 \alpha_{r} \tau}\right)}{2 \alpha_{r}} \\
\text { and } B(\gamma, \tau) & =\gamma d_{1} e^{-\alpha_{r} \tau} . \\
\text { Hence } g(t, r(t))^{1-\gamma} & =\exp [A(\gamma, T-t)+B(\gamma, T-t) r(t)+\gamma \psi(\gamma)(T-t)] .
\end{aligned}
$$

This implies that

$$
\begin{aligned}
V(t, x, r) & =\frac{1}{\gamma} e^{A(\gamma, T-t)+\gamma \psi(\gamma)(T-t)} e^{B(\gamma, T-t) r}(x+\pi f(t))^{\gamma} \\
\Rightarrow \theta_{A}(t, x, r) & =-\frac{\pi f(t)}{x}+\frac{(x+\pi f(t))}{x} \frac{\gamma}{(\gamma-1)}\left(1-e^{-\alpha_{r}(T-t)}\right) \\
\theta_{B}(t, x, r) & =\frac{(x+\pi f(t))}{x} \frac{\gamma e^{-\alpha_{r}(T-t)}}{(\gamma-1)} \\
\text { and } \theta_{C}(t, x, r) & =\frac{(x+\pi f(t))}{x} \frac{1}{(1-\gamma)} .
\end{aligned}
$$

These equations give us explicit formulae for the stochastic lifestyle strategy. In (3.4.7), $\theta_{A}(t, x, r)$ has been written in a way which highlights the two components to investments in $p_{A}$. First, we have a short holding of $-\pi f(t)$ which will be paid off precisely by the future premiums (since we have a complete market). Second, we have the augmented wealth $X(t)+\pi f(t)$ which is invested in fixed proportions in $p_{A}, p_{B}$ and $p_{C}$ which vary with the term to maturity only ${ }^{21} 22$.

\footnotetext{
${ }^{21}$ We note that none of the portfolio weights $\theta_{A}(t, x, r), \theta_{B}(t, x, r)$ and $\theta_{C}(t, x, r)$ depends upon $r$. This is a consequence of the choice of the Vasicek model and the simple form for $a(t, r(t))$. In other cases the $\theta$ 's will depend upon $r$ as well as on $t$.

${ }^{22}$ We can note here a similarity to the concept of portfolio insurance (see, for example, Black and Jones, 1988, Black and Perold, 1992, and Cairns, 2000). Portfolio insurance is an investment
} 
To complete the numerical example we note from equation (3.4.1) that:

$$
\begin{aligned}
f(t) & =\int_{t}^{T} \exp \left\{-\sigma_{Y}^{\prime} \xi(s-t)\right\} d s=\int_{t}^{T} \exp [-0.01(s-t)] d s \\
& =\frac{1-e^{-0.01(T-t)}}{0.01} .
\end{aligned}
$$

Examples of five scenarios are plotted in Figure 5 for this parameter set, together with $\pi=0.1$ and $\gamma=-5(R R A=6)$. The top graph in this figure shows the wealth-income ratio, $X(t)$, while the middle graph shows the replacement ratio that could be achieved with the current fund, current income and current annuity rates (that is, $X(t) / a(t, r(t))$ ). The five paths in the top two graphs give us an indication of the general spread of results. The minimum admissible fund size for the avoidance of insolvency $(-\pi f(t))$ is also plotted for reference and we can see that the actual fund size is always comfortably above this at all times on all sample paths. Compared with the top graph, the five paths in the middle graph are less spread out and smoother as the maturity date approaches because annuity risk is being hedged.

This observation is clearer in Figure 6 where we consider an extremely risk averse plan member. Since the market is complete in this case, the plan member is both willing and able to target a specific replacement ratio with certainty. This means that in the middle graph the sample replacement ratios, $X(t) / a(t, r(t))$, all converge to the same point at $T=20$. At intermediate times, $t$, they are more spread out because the investment strategy is targeting for certainty at $T=20$ rather than $t<T$. This plot demonstrates the importance of 'seeing the strategy through to its conclusion'. In the top graph in Figure 6 the tightness of the sample paths up to $t=10$ reflects the dominance of Fund A in the asset allocation strategy (as shown in the bottom graph). It is only when the strategy switches to Fund B that $X(t)$ starts to show significant variability. This is because Fund B is risky relative to the salary numeraire, $Y(t)$. In contrast, when risk aversion is low, then $X(t) / a(t, r(t))$ does not converge as $t \rightarrow T$ (Figure 5 middle graph).

In the bottom graph in Figure 5 we have selected the bold scenario in the upper graphs in order to show how the asset allocation strategy varies over time. The stochastic-lifestyling nature of the strategy is evident. Initially, when $X(t)$ is small there is considerable short-selling of the cash fund A (in other words borrowing cash) in anticipation of future premiums. Also for small $t$ the asset mix shows a fair degree of randomness (which is different for each of the 5 scenarios). This is

strategy that aims to ensure that the value of a pension plan never falls below a specified floor. Here the floor of 0 at time $T$ imposes a floor of $-\pi f(t)$ at time $t$. Furthermore, the form of the utility function at $T$ dictates the way that we invest the surplus over the floor. Since the value of the floor is negative, in contrast to the traditional positive floor in portfolio insurance, greater net pension wealth implies a lower proportion invested in equities. 
because the asset mix is most sensitive to changes in $x$ when $x$ is small, which will usually be when $t$ is also small. Later on the asset mix seems to follow a relatively smooth path, when $X(t)$ is larger. For clarity, only one sample path has been plotted in the bottom graph. When we plot several sample paths, say, for $\theta_{C}(t)$ we would find that the individual paths can be quite different early on in the contract ${ }^{23}$. However, the sample paths $\theta_{C}(t)$ all converge to the same limiting value.

\subsubsection{The cost of suboptimality}

Up until now we have focused on the derivation and analysis of the optimal stochastic asset-allocation strategy. So we have worked our way through the theory. Now we have to ask ourselves: is it worth our whiles trying to implement such a strategy in practice? Rephrasing this question: does the use of the optimal stochastic lifestyle strategy significantly improve the welfare of a plan member relative to the more commonly used DC strategies? If it turns out that stochastic lifestyling does not improve welfare significantly then the effort of trying to implement it might not be worthwhile. In contrast, if we find that stochastic lifestyling does add significant value then we should be trying to persuade pension providers to consider alternative strategies that are closer to stochastic lifestyling than the current range of strategies that are typically offered.

In Table 1 we give numerical results for different levels of risk aversion $(R R A=$ 1, 6, 12 corresponding to $\left.{ }^{24} \gamma=0,-5,-11\right)$ and policy durations $(T=20,40$ years). For each $(\gamma, T)$ combination we have considered seven asset allocation strategies:

- Optimal stochastic lifestyle strategy, $p^{*}(t, X(t), r(t))$.

- Salary-hedged static strategy (S). This is the strategy

$$
p(t, X(t), r(t))=\frac{-\gamma}{1-\gamma} p_{B}+\frac{\gamma}{1-\gamma} p_{C} \quad \text { for all }(t, X(t), r(t)) .
$$

This is the limiting value of $p^{*}(t, X(t), r(t))$ as $t \rightarrow T$ and takes account of the correlation between salaries and the risky assets as well as the conversion of pension wealth into an annuity at $T$.

- Merton-static strategy (M). This is the static strategy

$$
p(t, X(t), r(t))=\frac{\gamma}{1-\gamma} p_{C} \quad \text { for all }(t, X(t), r(t)) .
$$

\footnotetext{
${ }^{23}$ For example, for the 5 sample paths plotted in Figure 5 (top and middle) $\theta_{C}(5)$ ranged from 0.44 to 0.78 .

${ }^{24}$ We actually set $\gamma=+0.01$ which gives essentially the same results as for $\gamma=0$, but is computationally more convenient.
} 


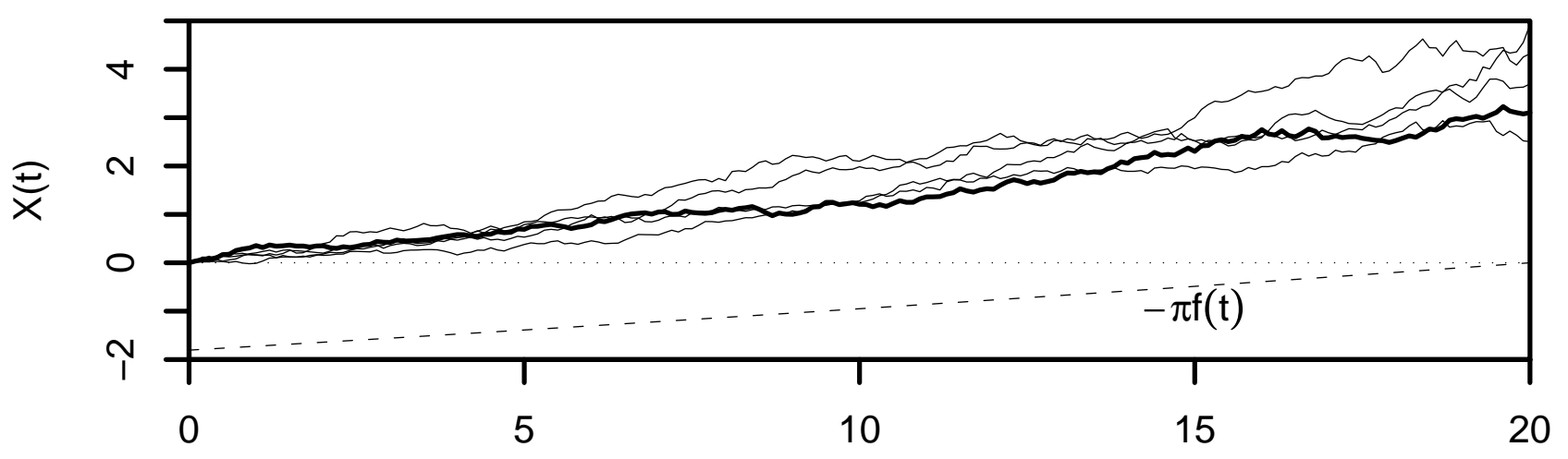

Time, $\mathrm{t}$
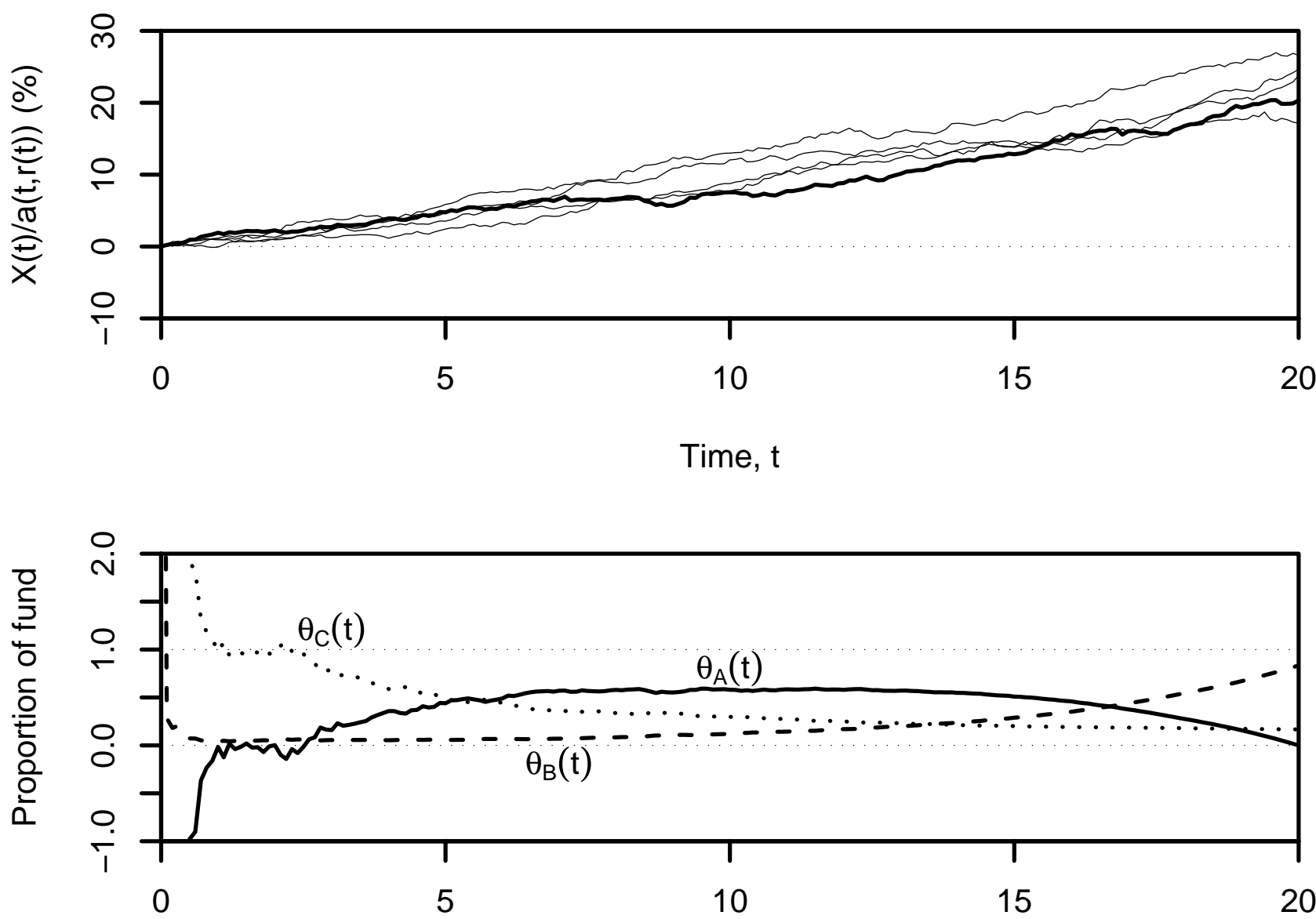

Time, $\mathrm{t}$

Figure 5: Dynamics of a plan member's pension wealth over $0<t<T=20$ for relative risk aversion of $6(\gamma=-5)$, and $\pi=0.1$. Top: $X(t)=W(t) / Y(t)$; 5 scenarios (solid lines) with the minimum $-\pi f(t)$ (dashed line). Middle: $X(t) / a(t, r(t))=W(t) / Y(t) a(t, r(t))$; same 5 scenarios (solid lines). Bottom: proportions invested in portfolios $p_{A}, p_{B}$ and $p_{C}: \theta_{A}(t)$ (solid line), $\theta_{B}(t)$ (dashed line) and $\theta_{C}(t)$ (dotted line) corresponding to the bold scenario in the top and middle graphs. 


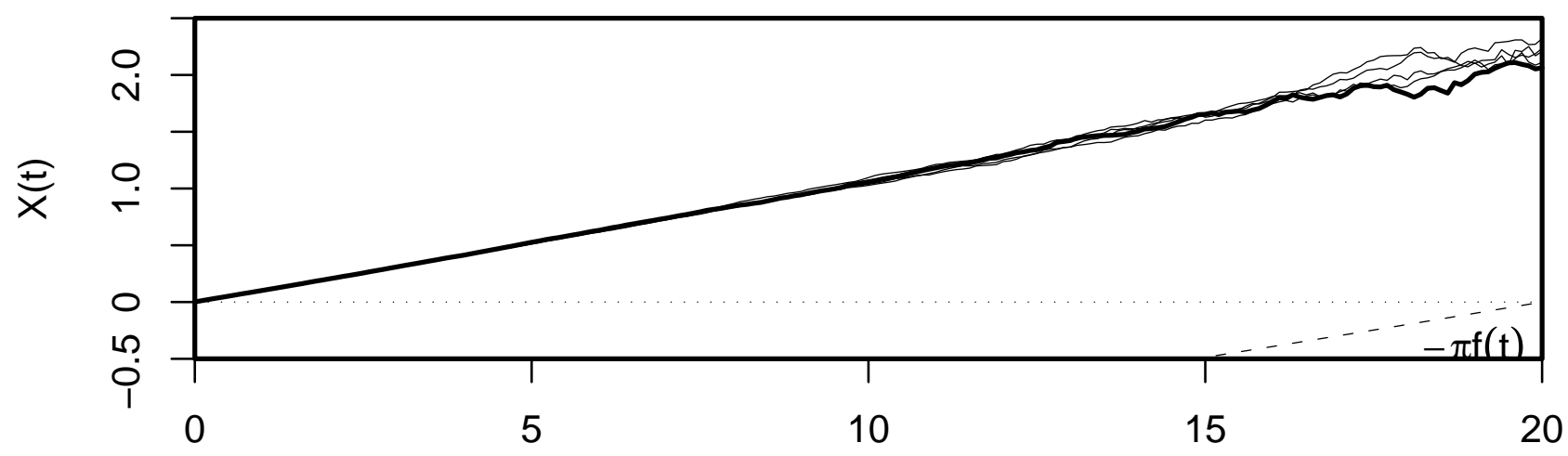

Time, $\mathrm{t}$

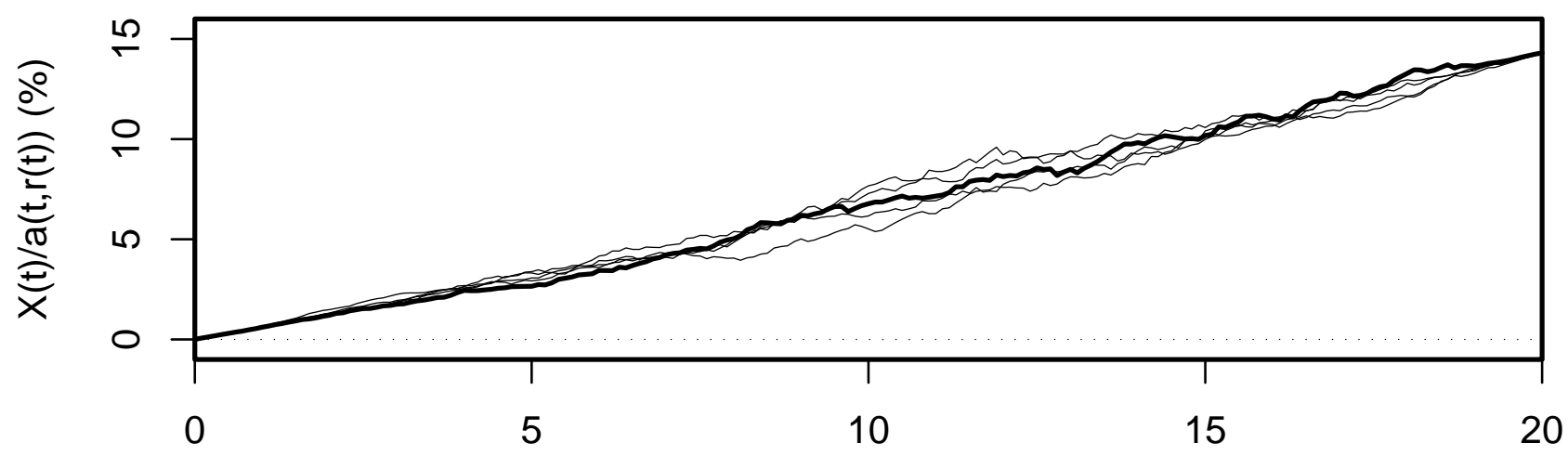

Time, $\mathrm{t}$

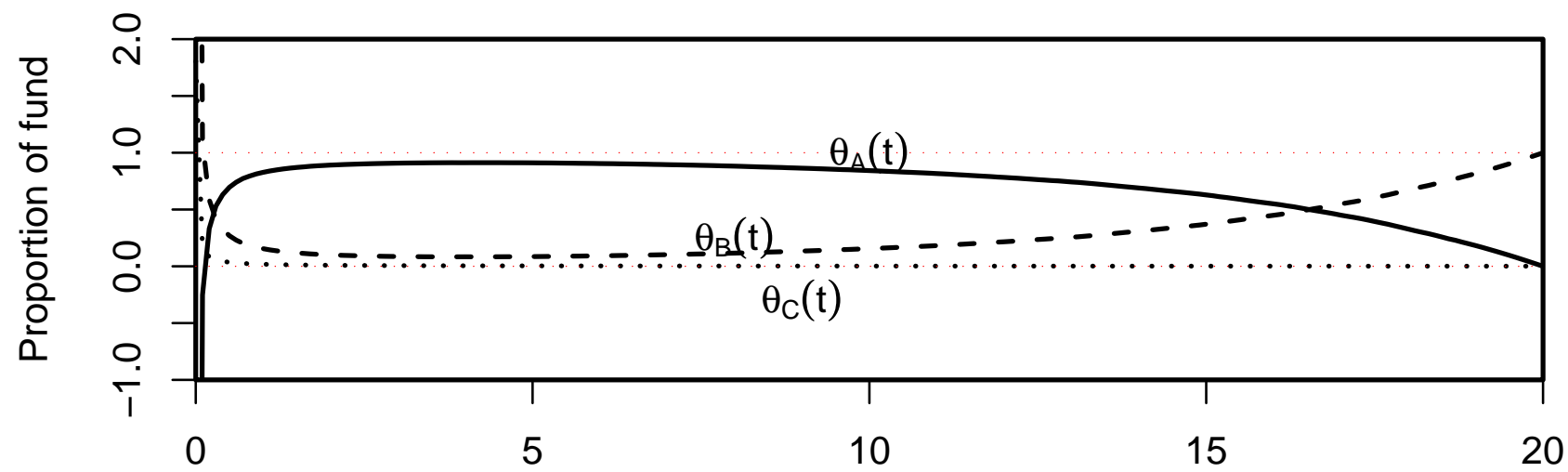

Time, $\mathrm{t}$

Figure 6: Dynamics of a plan member's pension wealth over $0<t<T=20$ for relative risk aversion $1001(\gamma=-1000)$, and $\pi=0.1$. Top: $X(t)=W(t) / Y(t)$; 5 scenarios (solid lines) with the minimum $-\pi f(t)$ (dashed line). Middle: $X(t) / a(t, r(t))=W(t) / Y(t) a(t, r(t))$; same 5 scenarios (solid lines). Bottom: proportions invested in portfolios $p_{A}, p_{B}$ and $p_{C}: \theta_{A}(t)$ (solid line), $\theta_{B}(t)$ (dashed line) and $\theta_{C}(t)$ (dotted line) corresponding to the bold scenario in the top and middle graphs. 
This is the classical Merton strategy which does take account of relative risk aversion, $R R A=1-\gamma$, but does not make allowance for correlation between salary and the asset returns, or the pension conversion.

- Deterministic lifestyle strategy. The general lifestyle strategy invests $100 \%$ in equities (Fund C) until $\tau=10$ or 5 years before retirement. Over the final $\tau$, years the equity investments are gradually switched wholly into bonds (Fund B) or wholly into cash (Fund A). The four strategies are labelled B for a switch into Fund B or A for Fund A; and by the length of the switching period (5 or 10 years).

In each sub-table ((a) to (f)) there are two rows of numbers. In the first row, we give the expected terminal utility. For convenience these have been rescaled so that the optimal value is +100 or -100 (depending on whether the raw value is positive or negative). This row only allows us to rank the different strategies. The numbers give us no indication of how much of an improvement in welfare a plan member will get as a result of using the optimal stochastic strategy. In the second row, therefore, we give a more concrete measure of the cost of adopting the suboptimal strategy relative to the optimal one. For example, in Table 1 (a) the static strategy $\mathrm{S}$ has a cost of $37.9 \%$ of the original contribution rate of 10\% of salary: that is, the plan member would have to pay a contribution rate of $\pi^{\prime}=\pi \times 1.379$ or $13.79 \%$ of salary to get the same expected terminal utility as the theoretical optimum. ${ }^{25}$

We can make the following observations:

- The cost is higher for 40-year time horizons than for 20-year horizons. This reflects various interacting factors. However, the main reason is simply that the longer the duration of suboptimal investment the greater the the costs of suboptimality.

- The costs of suboptimality vary substantially as we move from one level of risk aversion to another but often vary in a non-monotonic way. For example, strategy B-5 with $T=40$ has costs of $196.7 \%, 40.4 \%$ and $97.2 \%$ of the original contribution rate for members with RRA's of 1,6 and 12 respectively. There are two reasons for this variation with the RRA. First, the deterministic strategy might give a reasonably good approximation to $p^{*}(t, x)$ for certain values (e.g. medium) of $\gamma$. The more accurate the approximation the lower the cost of suboptimality. (For example, static strategy $\mathrm{S}$

\footnotetext{
${ }^{25}$ To place this on a more theoretical foundation: the plan sponsor would have to increase this member's salary from $Y(t)$ to $\bar{Y}(t)=1.0379 Y(t)$ and the contribution rate would be $\bar{\pi}=$ $0.1379 / 1.0379$ or $13.29 \%$ of the revised salary, $\bar{Y}(t)$. Under this revised scheme the pre-retirement consumption rate $(1-\bar{\pi}) \bar{Y}(t)=(1-\pi) Y(t)$ is unaltered.
} 


\begin{tabular}{|l|c|c|c|c|c|c|c|}
\hline (a) & \multicolumn{6}{|c|}{$\gamma=0(R R A=1), \quad T=20$} \\
\hline Strategy: & Optimal & \multicolumn{2}{|c|}{ Static } & \multicolumn{4}{c|}{ Deterministic lifestyle } \\
& stochastic & $\mathrm{S}$ & $\mathrm{M}$ & $\mathrm{B}-10$ & $\mathrm{~B}-5$ & $\mathrm{~A}-10$ & $\mathrm{~A}-5$ \\
\hline$V(0,0)$ & 100 & 99.68 & 99.68 & 99.30 & 99.38 & 99.24 & 99.35 \\
\hline Cost & $0 \%$ & $37.9 \%$ & $37.8 \%$ & $101.8 \%$ & $86.7 \%$ & $113.9 \%$ & $92.3 \%$ \\
\hline
\end{tabular}

\begin{tabular}{|l|c|c|c|c|c|c|c|}
\hline (b) & \multicolumn{7}{|c|}{$\gamma=0(R R A=1), \quad T=40$} \\
\hline Strategy: & Optimal & \multicolumn{2}{|c|}{ Static } & \multicolumn{5}{c|}{ Deterministic lifestyle } \\
& stochastic & $\mathrm{S}$ & $\mathrm{M}$ & $\mathrm{B}-10$ & $\mathrm{~B}-5$ & $\mathrm{~A}-10$ & $\mathrm{~A}-5$ \\
\hline$V(0,0)$ & 100 & 99.45 & 99.45 & 98.84 & 98.92 & 98.77 & 98.89 \\
\hline Cost & $0 \%$ & $73.7 \%$ & $73.6 \%$ & $222.1 \%$ & $196.7 \%$ & $243.3 \%$ & $206.4 \%$ \\
\hline
\end{tabular}

\begin{tabular}{|l|c|c|c|c|c|c|c|}
\hline$(\mathrm{c})$ & \multicolumn{6}{|c|}{$\gamma=-5(R R A=6), \quad T=20$} \\
\hline Strategy: & Optimal & \multicolumn{2}{|c|}{ Static } & \multicolumn{4}{c|}{ Deterministic lifestyle } \\
& stochastic & $\mathrm{S}$ & $\mathrm{M}$ & $\mathrm{B}-10$ & $\mathrm{~B}-5$ & $\mathrm{~A}-10$ & $\mathrm{~A}-5$ \\
\hline$V(0,0)$ & -100 & -134.58 & -205.42 & -141.00 & -194.01 & -191.47 & -236.86 \\
\hline Cost & $0 \%$ & $6.1 \%$ & $15.5 \%$ & $7.1 \%$ & $14.2 \%$ & $13.9 \%$ & $18.8 \%$ \\
\hline
\end{tabular}

\begin{tabular}{|l|c|c|c|c|c|c|c|}
\hline$(\mathrm{d})$ & \multicolumn{6}{|c|}{$\gamma=-5(R R A=6), \quad T=40$} \\
\hline Strategy: & Optimal & \multicolumn{2}{|c|}{ Static } & \multicolumn{4}{c|}{ Deterministic lifestyle } \\
& stochastic & $\mathrm{S}$ & $\mathrm{M}$ & $\mathrm{B}-10$ & $\mathrm{~B}-5$ & $\mathrm{~A}-10$ & $\mathrm{~A}-5$ \\
\hline$V(0,0)$ & -100 & -202.92 & -314.64 & -351.06 & -545.40 & -477.53 & -682.83 \\
\hline Cost & $0 \%$ & $15.2 \%$ & $25.8 \%$ & $28.6 \%$ & $40.4 \%$ & $36.7 \%$ & $46.8 \%$ \\
\hline
\end{tabular}

\begin{tabular}{|l|c|c|c|c|c|c|c|}
\hline$(\mathrm{e})$ & \multicolumn{6}{|c|}{$\gamma=-11(R R A=12), T=20$} \\
\hline Strategy: & Optimal & \multicolumn{2}{|c|}{ Static } & \multicolumn{4}{c|}{ Deterministic lifestyle } \\
& stochastic & $\mathrm{S}$ & $\mathrm{M}$ & $\mathrm{B}-10$ & $\mathrm{~B}-5$ & $\mathrm{~A}-10$ & $\mathrm{~A}-5$ \\
\hline$V(0,0)$ & -100 & -192.4 & -800.9 & -562.1 & -3379.7 & -1326.5 & -5519.4 \\
\hline Cost & $0 \%$ & $6.1 \%$ & $20.8 \%$ & $17 \%$ & $37.7 \%$ & $26.5 \%$ & $44 \%$ \\
\hline
\end{tabular}

\begin{tabular}{|l|c|c|c|c|c|c|c|}
\hline$(\mathrm{f})$ & \multicolumn{7}{|c|}{$\gamma=-11(R R A=12), \quad T=40$} \\
\hline Strategy: & Optimal & \multicolumn{2}{|c|}{ Static } & \multicolumn{5}{c|}{ Deterministic lifestyle } \\
& stochastic & $\mathrm{S}$ & $\mathrm{M}$ & $\mathrm{B}-10$ & $\mathrm{~B}-5$ & $\mathrm{~A}-10$ & $\mathrm{~A}-5$ \\
\hline$V(0,0)$ & -100 & -1048 & -2066 & -25860 & -175732 & -57602 & -309662 \\
\hline Cost & $0 \%$ & $23.8 \%$ & $31.7 \%$ & $65.7 \%$ & $97.2 \%$ & $78.2 \%$ & $107.7 \%$ \\
\hline
\end{tabular}

Table 1: Relative expected terminal utility for different values of $\gamma$ and duration of contract, $T$. Expected terminal utilities have been normalised so that the optimal stochastic lifestyle strategy is +100 or -100 (for $\gamma \geq 0$ or $\gamma<0$ respectively). Cost is the cost of suboptimality: the relative increase required in the contribution rate to match the optimal expected terminal utility assuming a contribution rate of $10 \%$ of salary. 
always gives a better approximation than the determinstic lifestyle strategies.) Second, the general increases in the cost of suboptimality for members with $R R A=12$ reflect the more severe penalties that apply when there is even a small deviation from the optimal strategy.

- When members have $R R A=1$, the static strategies $\mathrm{S}$ and $\mathrm{M}$ are almost identical, so that the costs are almost equal.

- When members have $R R A=1$, the costs of suboptimality are highly significant. One reason for this is that the stochastic lifestyling strategy varies substantially over time, so that even the best, unconstrained static strategy looks very poor. Moreover, the low RRA implies that it is optimal most of the time to be very short in cash (even strategies $\mathrm{S}$ and $\mathrm{M}$ are $-100 \%$ of the net pension wealth in cash). Deterministic lifestyle strategies are therefore very costly because they implicitly have a no-short-selling constraint. ${ }^{26}$

- When members have $R R A=12$, determinstic lifestyle strategies are also very poor. The reason is that the initial $100 \%$ equity investment is far too high for such highly risk-averse individuals.

- When members have the intermediate value of $R R A=6$, we see that the costs of suboptimality are not so great, although they are not negligible either. This is not because of any special characteristic of such members. It is simply that the reasons discussed above explaining why the sub-optimal strategies are so costly for members with both lower and higher $R R A$ 's do not apply so strongly for members with $R R A=6$.

- The salary-hedged static strategy, S, always outperforms significantly the deterministic lifestyle strategies. This indicates the importance of taking into account the plan member's personal salary profile rather than selling a standard 'one-size-fits-all' asset allocation strategy.

- The B-5 and B-10 strategies are all superior to the corresponding A-5 and A-10 strategies. This indicates that the plan member's preference for a pension at retirement over a cash lump sum needs to be matched by a switch into long-dated bonds before retirement (B strategies) rather than cash (A strategies).

- Finally, we note that the determinstic lifestyle strategies are the worst strategies of all.

\footnotetext{
${ }^{26}$ This constraint can be mitigated somewhat by permitting the use of highly-geared mutual funds: an investment of $200 \%$ of net pension wealth in equities (which is not permissible) (and $-100 \%$ in cash) might then be equivalent, say, to a $100 \%$ investment in a highly-geared fund (which is permissible).
} 


\section{Conclusions}

Stochastic lifestyling has at least two advantages over deterministic lifestyling in respect of defined contribution pension plans: it takes into account both the plan member's attitude to risk and the correlation between his salary and asset returns. It is implemented using three efficient mutual funds, resembling investment in cash, bonds and equities, respectively. The equity fund is regarded as high risk and its purpose is to satisfy the risk appetite of the plan member. The cash and bond funds are regarded as low risk, but serve different purposes: in particular, the former can be interpreted as a default low-risk investment, whereas the latter is a hedge against annuity-rate risk. In the early stages of the plan, the cash fund is therefore the dominant low-risk component of the investment strategy, but as the retirement date approaches, there is a gradual switch from cash into bonds. This switching between mutual funds superficially resembles deterministic lifestyling. However, instead of switching from high-risk assets to low-risk assets, as in the case of deterministic lifestyling, the optimal stochastic lifestyle strategy involves a switch between different types of low-risk assets.

Our results have important practical relevance, since they suggest that the costs of suboptimal policies can be considerable: plan members can (typically) expect to be substantially better off if they adopt a stochastic lifestyling strategy rather than a either a static or a deterministic-lifestyling asset-allocation strategy. Our results also suggest that many commercial pension plans could be significantly improved by making appropriate use of cash and bond investments along stochasticlifestyling lines. If the investment opportunity set and the plan member's attitude to risk are unchanging, all but two of the parameters needed to operate the strategy are predetermined and the values of the two stochastic variables required (namely the ratio of current pension wealth to the plan member's current salary and the risk-free rate of interest) are easy to measure.

Finally, the following extensions to the current study suggest themselves: (a) developing numerical results for Case 4 with stochastic interest rates, although we conjecture that the results will be similar to those reported in Table 1, (b) allowing for a more general model determining salary growth, e.g., letting $\mu_{Y}(t)$ be stochastic, and (c) considering more general utility functions, such as Epstein \& Zin (1989).

\section{Acknowledgements}

The authors gratefully acknowledge support from the BSI Gamma Foundation. They also wish to thank participants at the "Risk Theory" and "Stochastic Analysis in Finance and Insurance" meetings in Oberwolfach in September 1999 and 
May 2000 respectively, for their helpful comments. This includes, in particular, Griselda Deelstra and Ralf Korn. Finally, they wish to thank the two referees of the paper for their extremely helpful comments.

\section{References}

Ando, A., and Modigliani, F. (1963) The 'life cycle' hypothesis of saving: Aggregate implications and tests. American Economic Review 53: 55-84.

Björk, T. (1998) Arbitrage Theory in Continuous Time. OUP, Oxford.

Black, F., and Jones, R. (1988) Simplifying portfolio insurance for corporate pension plans. Journal of Portfolio Management, 14: 33-37.

Black, F., and Perold, A. (1992) Theory of constant proportion portfolio insurance. Journal of Economic Dynamics and Control, 16: 403-426.

Blake, D., Cairns, A.J.G., and Dowd, K. (2001) PensionMetrics I: Stochastic pension plan design and value at risk during the accumulation phase. Insurance: Mathematics and Economics 29: 187-215.

Boulier, J-F., Huang, S-J., and Taillard, G. (2001) Optimal management under stochastic interest rates: The case of a protected pension fund. Insurance: Mathematics and Economics 28: 173-189.

Cairns, A.J.G. (2000) Some notes on the dynamics and optimal control of stochastic pension fund models in continuous time. ASTIN Bulletin, 30: 19-55.

Constantinides, G.M. (1990) Habit formation: a resolution of the equity premium puzzle. The Journal of Political Economy, 98: 519-543.

Cox, J.C., and Huang, C-F. (1991) A variational problem arising in financial economics. Journal of Mathematical Economics 20:465-487.

Deelstra, G., Grasselli, M., and Koehl, P-F. (2000) Optimal investment strategies in a CIR framework. Journal of Applied Probability 37:936-946.

Duffie, D., Fleming, W., Soner, H.M., and Zariphopoulou, T. (1997) Hedging in incomplete markets with HARA utility. Journal of Economic Dynamics and Control 21: 753-782.

Epstein, L.G., and Zin, S.E. (1989) Substitution, risk aversion, and the temporal behavior of consumption and asset returns: A theoretical framework. Econometrica, 57: 937-969.

Karatzas, I., Lehoczky, J.P., and Shreve, S.E. (1987) Optimal portfolio and consumption decisions for a small investor on a finite horizon. SIAM Journal of Control and Optimization 25: 1557-1586. 
Korn, R. (1997) Optimal Portfolios. World Scientific, Singapore.

Korn, R., and Krekel, M. (2002) Optimal portfolios with fixed consumption and income streams. Working Paper, University of Kaiserslautern.

Liu, J. (2001) Portfolio selection in stochastic environments. Working Paper, Anderson School of Management, UCLA.

Malkiel, B.G. (2003) A Random Walk Down Wall Street. Norton, New York.

Merton, R.C. (1969) Lifetime portfolio selection under uncertainty: The continuous-time case. Review of Economics and Statistics 51: 247-257.

Merton, R.C. (1971) Optimum consumption and portfolio rules in a continuoustime model. Journal of Economic Theory 3: 373-413.

Merton, R.C. (1990) Continuous-Time Finance. Blackwell, Cambridge, Mass.

Øksendal, B. (1998) Stochastic Differential Equations, 5th Edition. SpringerVerlag, Berlin.

Ryder, H.E., and Heal, G.M. (1973) Optimum growth with intertemporally dependent preferences. Review of Economic Studies, 40: 1-33.

Sundaresan, S.M. (1989) Intertemporally dependent preferences and the volatility of consumption and wealth. Review of Financial Studies, 2: 73-89.

Sundaresan, S., and Zapatero, F. (1997) Valuation, optimal asset allocation and retirement incentives of pension plans. Review of Financial Studies, 10: 631-660. Vasicek, O.E. (1977) An equilibrium characterisation of the term structure. Journal of Financial Economics 5: 177-188. 


\section{Appendices}

\section{A Proof of Theorem 3.2.1}

Let us consider the composition of $p^{*}(t, x, r ; V)$ in equation (3.2.2) in terms of three efficient portfolios or mutual funds which we denote $p_{A}, p_{B}$, and $p_{C}$.

Suppose that $\pi=0$. Then $d X(t) / X(t)$ has an expected value of $m(p)=p^{\prime} C(\xi-$ $\left.\sigma_{Y}\right)-\tilde{\mu}_{Y}(t)$ and an instantaneous variance of $v(p)=\sigma_{Y 0}^{2}+\sigma_{Y}^{\prime} \sigma_{Y}+p^{\prime} D p-2 p^{\prime} C \sigma_{Y}$, where $D=C C^{\prime}$. Now minimise $v(p)$ over $p$ :

$$
\begin{aligned}
\Rightarrow 2 D p-2 C \sigma_{Y} & =0 \\
\Rightarrow p^{*}(0) & =D^{-1} C \sigma_{Y}=C^{\prime-1} \sigma_{Y} \equiv p_{A}, \text { say. } \\
\text { Then } m\left(p_{A}\right) & =\sigma_{Y}^{\prime}\left(\rho-\sigma_{Y}\right)-\tilde{\mu}_{Y}(t) \equiv m_{A}, \text { say. }
\end{aligned}
$$

Next minimise $v(p)$ over $p$ subject to $m(p)=m$, using the method of Lagrange multipliers, with $L(p, \psi)=v(p)+2 \psi(m(p)-m)$. Therefore,

$$
\begin{aligned}
\frac{\partial L}{\partial p} & =2 D p-2 C \sigma_{Y}+2 \psi C\left(\xi-\sigma_{Y}\right)=0 \\
\Rightarrow p^{*}\left(m-m_{A}\right) & =C^{\prime-1}\left[(1+\psi) \sigma_{Y}-\psi \xi\right] \\
\frac{\partial L}{\partial \psi} & =2(m(p)-m)=0 \\
\Rightarrow 0 & =\left[C \sigma_{Y}-\psi C\left(\xi-\sigma_{Y}\right)\right]^{\prime} D^{-1} C\left(\xi-\sigma_{Y}\right)-\tilde{\mu}_{Y}(t)-m \\
\Rightarrow \psi & =\psi(m)=\frac{m_{A}-m}{\left(\xi-\sigma_{Y}\right)^{\prime}\left(\xi-\sigma_{Y}\right)} .
\end{aligned}
$$

The important point to note is that the optimal portfolio $p^{*}\left(m-m_{A}\right)$ is a weighted average of the minimum-variance portfolio $p_{A}=C^{\prime-1} \sigma_{Y}$ (with weight $1+\psi$ ) and another efficient portfolio $p_{C}=C^{\prime-1} \xi$ (with weight $-\psi$ ).

Now consider $H(t)=X(t) / a(t, r(t))$ where $a(t, r(t))$ is defined in equation (3.1.1). Since $a(t, r(t))$ is a function of $r(t)$ only, we can write (using Ito's formula) $d a(t, r(t))$ as $a(t, r(t))\left[-d_{a}(r) d r(t)+\frac{1}{2} c_{a}(r)(d r(t))^{2}\right]$, where $d_{a}(r)$ is the duration of the annuity function and $c_{a}(r)$ is its convexity,

$$
\begin{aligned}
d_{a}(r) & =-\frac{1}{a(t, r)} \frac{\partial a(t, r)}{\partial r} \\
\text { and } c_{a}(r) & =\frac{1}{a(t, r)} \frac{\partial^{2} a(t, r)}{\partial r^{2}} .
\end{aligned}
$$


Therefore,

$$
\begin{aligned}
d H(t)=H(t) & {\left[\left(p^{\prime} C\left(\xi-\sigma_{Y}\right)-\tilde{\mu}_{Y}(t)\right) d t-\sigma_{Y 0} d Z_{0}(t)+\left(p^{\prime} C-\sigma_{Y}^{\prime}\right) d Z(t)\right.} \\
+ & d_{a}(r)\left\{\mu_{r}(r) d t+\sigma_{r}(r)^{\prime} d Z(t)\right\} \\
& \left.+\left(d_{a}(r)^{2}-\frac{1}{2} c_{a}(r)\right) \sigma_{r}(r)^{\prime} \sigma_{r}(r) d t+d_{a}(r)\left(p^{\prime} C-\sigma_{Y}^{\prime}\right) \sigma_{r}(r) d t\right] \\
& \underline{\underline{\mathcal{D}}} \quad \\
& H(t)(m(p, r) d t+\sqrt{v(p, r)} d \tilde{Z}),
\end{aligned}
$$

where $\tilde{Z}(t)$ is a Brownian motion, $\underline{\mathcal{D}}$ means 'equivalent in distribution',

$$
\begin{gathered}
m(p, r)=p^{\prime} C\left(\xi-\sigma_{Y}\right)-\tilde{\mu}_{Y}(t)+d_{a}(r) \mu_{r}(r)+d_{a}(r)\left(p^{\prime} C-\sigma_{Y}^{\prime}\right) \sigma_{r}(r) \\
+\left(d_{a}(r)^{2}-\frac{1}{2} c_{a}(r)\right) \sigma_{r}(r)^{\prime} \sigma_{r}(r)
\end{gathered}
$$

and $v(p, r)=\sigma_{Y 0}^{2}+\sigma_{Y}^{\prime} \sigma_{Y}+p^{\prime} D p-2 p^{\prime} C \sigma_{Y}+2 d_{a}(r) p^{\prime} C \sigma_{r}(r)$

$$
-2 d_{a}(r) \sigma_{Y}^{\prime} \sigma_{r}(r)+d_{a}(r)^{2} \sigma_{r}(r)^{\prime} \sigma_{r}(r) \text {. }
$$

Now, minimise $v(p, r)$ over $p:^{27}$

$$
\begin{aligned}
\Rightarrow 2 D p-2 C \sigma_{Y}+2 d_{a}(r) C \sigma_{r}(r) & =0 \\
\Rightarrow p & =C^{\prime-1}\left(\sigma_{Y}-d_{a}(r) \sigma_{r}(r)\right)=p_{B}, \text { say. }
\end{aligned}
$$

Let $m_{B}=m\left(p_{B}, r\right)$. Next, minimise $v(p, r)$ over $p$ subject to $m(p, r)=m$. Let $p_{B}\left(m-m_{B}\right)$ be the optimal $p$ for this problem. Let $L(p, \psi)=v(p, r)+2 \psi(m(p, r)-$ $m)$.

Therefore,

$$
\begin{aligned}
\frac{\partial L}{\partial p} & =2 D p-2 C \sigma_{Y}+2 d_{a}(r) C \sigma_{r}(r)+2 \psi C\left(\xi-\sigma_{Y}+d_{a}(r) \sigma_{r}(r)\right)=0 \\
\Rightarrow p & =C^{\prime-1}\left(\sigma_{Y}-d_{a}(r) \sigma_{r}(r)-\psi\left(\xi-\sigma_{Y}+d_{a}(r) \sigma_{r}(r)\right)\right) \\
& =(1+\psi) C^{\prime-1}\left(\sigma_{Y}-d_{a}(r) \sigma_{r}(r)\right)-\psi C^{\prime-1} \xi \\
& =(1+\psi) p_{B}-\psi p_{C} \\
\frac{\partial L}{\partial \psi} & =0 \\
\Rightarrow \psi & =\frac{m_{B}-m}{\left(\xi-\sigma_{Y}+d_{a}(r) \sigma_{r}(r)\right)^{\prime}\left(\xi-\sigma_{Y}+d_{a}(r) \sigma_{r}(r)\right)} .
\end{aligned}
$$

${ }^{27}$ Note that $p_{B}=p_{A}$ if $d_{a}(r)=0$ (that is, if we are funding for a cash lump sum rather than a pension at retirement). 
As before we see that the optimal asset allocation strategy, $p_{B}\left(m_{B}-m, r\right)$, is a weighted average of the minimum variance portfolio, $p_{B}$, and the more risky, but still efficient, portfolio, $p_{C}$, derived earlier.

\section{B Proof of Theorem 3.3.1}

That $V(t, x, r)$ is proportional to $x^{\gamma}$ is clear. For example, suppose we replace $x$ by $k x$ but follow the same asset allocation strategy $p(t)$. We see that $J(t, k x, r, p)=$ $k^{\gamma} J(t, x, r, p)$ since $X(T)$ is replaced by $k X(T)$ if we follow the same strategy.

Now try $V=\gamma^{-1} g^{1-\gamma} x^{\gamma}$ in equation (3.3.1). First note that

$$
\begin{aligned}
V_{t} & =\frac{(1-\gamma)}{\gamma} g^{-\gamma} g_{t} x^{\gamma} \\
V_{x} & =g^{1-\gamma} x^{\gamma-1} \\
V_{x x} & =(\gamma-1) g^{1-\gamma} x^{\gamma-2} \\
V_{r} & =\frac{(1-\gamma)}{\gamma} g^{-\gamma} g_{r} x^{\gamma} \\
V_{r r} & =(\gamma-1) g^{-\gamma-1} g_{r}^{2} x^{\gamma}+\frac{(1-\gamma)}{\gamma} g^{-\gamma} g_{r r} x^{\gamma} \\
\text { and } V_{x r} & =(1-\gamma) g^{-\gamma} g_{r} x^{\gamma-1} .
\end{aligned}
$$

Then (3.2.3) becomes

$$
\begin{gathered}
\frac{(1-\gamma)}{\gamma} g^{-\gamma} g_{t} x^{\gamma}+\mu_{r}(r) \frac{(1-\gamma)}{\gamma} g^{-\gamma} g_{r} x^{\gamma} \\
+\left(-\tilde{\mu}_{Y}(t)+\sigma_{Y}^{\prime}\left(\xi-\sigma_{Y}\right)\right) g^{1-\gamma} x^{\gamma} \\
+\frac{1}{2} \sigma_{r}(r)^{\prime} \sigma_{r}(r)\left[(\gamma-1) g^{-\gamma-1} g_{r}^{2} x^{\gamma}+\frac{(1-\gamma)}{\gamma} g^{-\gamma} g_{r r} x^{\gamma}\right] \\
-\frac{1}{2}\left(\xi-\sigma_{Y}\right)^{\prime}\left(\xi-\sigma_{Y}\right) \frac{g^{2-2 \gamma} x^{2 \gamma-2}}{(\gamma-1) g^{1-\gamma} x^{\gamma-2}} \\
-\left(\xi-\sigma_{Y}\right) \sigma_{r}(r) \frac{g^{1-\gamma} x^{\gamma-1}(1-\gamma) g^{-\gamma} g_{r} x^{\gamma-1}}{(\gamma-1) g^{1-\gamma} x^{\gamma-2}} \\
-\frac{1}{2} \sigma_{r}(r)^{\prime} \sigma_{r}(r) \frac{(1-\gamma)^{2} g^{-2 \gamma} g_{r}^{2} x^{2 \gamma-2}}{(\gamma-1) g^{1-\gamma} x^{\gamma-2}}=0 .
\end{gathered}
$$


Now take out $g^{-\gamma-1} x^{\gamma}$ as a common factor. Then

$$
\begin{gathered}
\frac{(1-\gamma)}{\gamma} g g_{t}+\mu_{r}(r) \frac{(1-\gamma)}{\gamma} g g_{r}+\left(-\tilde{\mu}_{Y}(t)+\sigma_{Y}^{\prime}\left(\xi-\sigma_{Y}\right)\right) g^{2} \\
+\frac{1}{2} \sigma_{r}(r)^{\prime} \sigma_{r}(r)\left((\gamma-1) g_{r}^{2}+\frac{(1-\gamma)}{\gamma} g g_{r r}\right)-\frac{1}{2}\left(\xi-\sigma_{Y}\right)^{\prime}\left(\xi-\sigma_{Y}\right) \frac{1}{(\gamma-1)} g^{2} \\
+\left(\xi-\sigma_{Y}\right)^{\prime} \sigma_{r}(r) g g_{r}-\frac{1}{2}(\gamma-1) \sigma_{r}(r)^{\prime} \sigma_{r}(r) g_{r}^{2}=0 .
\end{gathered}
$$

The $g_{r}^{2}$ terms cancel, and, with further simplification and division by $g$, we get

$$
\begin{aligned}
\frac{(1-\gamma)}{\gamma} g_{t}+\frac{1}{2} & \frac{(1-\gamma)}{\gamma} \sigma_{r}(r)^{\prime} \sigma_{r}(r) g_{r r}+\left(\frac{(1-\gamma)}{\gamma} \mu_{r}(r)+\left(\xi-\sigma_{Y}\right)^{\prime} \sigma_{r}(r)\right) g_{r} \\
& +\left(-\tilde{\mu}_{Y}(t)+\sigma_{Y}^{\prime}\left(\xi-\sigma_{Y}\right)-\frac{1}{2}\left(\xi-\sigma_{Y}\right)^{\prime}\left(\xi-\sigma_{Y}\right) \frac{1}{(\gamma-1)}\right) g=0 .
\end{aligned}
$$

In the final step we replace $\tilde{\mu}_{Y}(t)$ by $\mu_{Y}(t)-\sigma_{Y}^{\prime} \sigma_{Y}$ and multiply by $\gamma /(1-\gamma)$ to get the PDE for $g$ in equation (3.3.1) in the Theorem. 